ARTICLE OPEN

\title{
Novel Antarctic yeast adapts to cold by switching energy metabolism and increasing small RNA synthesis
}

\author{
D. Touchette ${ }^{1}$, I. Altshuler (iD) ${ }^{1}$, C. Gostinčar (D) ${ }^{2,3}$, P. Zalar ${ }^{2}$, I. Raymond-Bouchard ${ }^{1}$, J. Zajc ${ }^{4}$, C. P. McKay ${ }^{5}$, N. Gunde-Cimerman ${ }^{2}$ and $^{2}$ \\ L. G. Whyte (iD) ${ }^{1 凶}$
}

The Author(s) 2021

The novel extremophilic yeast Rhodotorula frigidialcoholis, formerly $R$. JG1b, was isolated from ice-cemented permafrost in University Valley (Antarctic), one of coldest and driest environments on Earth. Phenotypic and phylogenetic analyses classified $R$. frigidialcoholis as a novel species. To characterize its cold-adaptive strategies, we performed mRNA and sRNA transcriptomic analyses, phenotypic profiling, and assessed ethanol production at 0 and $23^{\circ} \mathrm{C}$. Downregulation of the ETC and citrate cycle genes, overexpression of fermentation and pentose phosphate pathways genes, growth without reduction of tetrazolium dye, and our discovery of ethanol production at $0{ }^{\circ} \mathrm{C}$ indicate that $R$. frigidialcoholis induces a metabolic switch from respiration to ethanol fermentation as adaptation in Antarctic permafrost. This is the first report of microbial ethanol fermentation utilized as the major energy pathway in response to cold and the coldest temperature reported for natural ethanol production. $R$. frigidialcoholis increased its diversity and abundance of sRNAs when grown at 0 versus $23^{\circ} \mathrm{C}$. This was consistent with increase in transcription of Dicer, a key protein for sRNA processing. Our results strongly imply that post-transcriptional regulation of gene expression and mRNA silencing may be a novel evolutionary fungal adaptation in the cryosphere.

The ISME Journal (2022) 16:221-232; https://doi.org/10.1038/s41396-021-01030-9

\section{INTRODUCTION}

The majority of the Earth's biosphere exists at permanently cold temperatures, below $5^{\circ} \mathrm{C}$, and includes multiple cryoenvironments $\left(<0^{\circ} \mathrm{C}\right)$, many of which are characterized by some of the most dry, low biomass, cold, and salty conditions on Earth. Despite these harsh conditions, microorganisms capable of metabolic activity and even growth in situ have been reported in these habitats and include bacteria, archaea, algae, fungi [1-3]. These cold-adapted microorganisms are termed as psychrophilic (optimum temperature below $15^{\circ} \mathrm{C}$ ) or psychrotolerant (optimum above $15^{\circ} \mathrm{C}$ ) [4], and are able to maintain viability for thousands of years in glacial ice $[5,6]$. Microbial communities in these environments have to overcome numerous biochemical and physiological challenges, including low water and nutrient availability, high oxidative stress, high solar irradiation, and multiple freeze-thaw cycles, coupled with a major decrease in membrane fluidity, enzymatic activity, and protein folding, and with the creation of stable secondary inhibitory DNA/RNA structures [4, 7-9].

While numerous studies have investigated bacteria, archaea and algae from Arctic and Antarctica environments [4, 10, 11], there has been a recent interest in fungi inhabiting these environments [12-14]. Many fungal species have been isolated and characterized from a diversity of extreme environments, such as brines [15], Arctic glaciers, and Antarctica rocks and deserts $[5,16,17]$. Fungi play key roles in the cryosphere as they are important facilitators of primary biomass production through endophytic and lichenic relationships $[18,19]$ and are involved in the nutrients recycling [20]. Although many basidiomycetous yeasts are adapted to low temperatures and detected in a broad range of cold ecosystems [21, 22], their adaptation strategies to low temperatures are not fully understood. Molecular mechanisms enabling yeast survival include the production of antifreeze and cold-active proteins, compatible solutes, and an increase in membrane fluidity $[5,23]$.

For example, the heterotrophic Rhodotorula yeasts are unicellular and pink-pigmented [24, 25] and have been isolated in many cold habitats [26]; numerous cold-adapted species, including $R$. aurantiaca, R. psychrophila, R. psychrophenolica, R. glacialis, and $R$. himalayensis $[27,28]$ have been identified. More recently, we isolated the putative novel psychrotolerant Rhodotorula JG1b strain from $~ 150,000$-year-old ice-cemented permafrost soil from University Valley, in the McMurdo Dry Valleys of Antarctica [24], one of the coldest and driest places on Earth [29]. Rhodotorula JG1b was one of only six microorganisms isolated and sequenced form this ice-cemented permafrost. It was capable of growth at temperatures as low as $-10^{\circ} \mathrm{C}$ [1] and tolerated up to $15 \% \mathrm{NaCl}$ and $12 \%$ perchlorate [24].

In this study, Rhodotorula JG1 was used as a model yeast to determine its adaptations to cold temperatures and how it survives in one of the coldest environments on Earth. Our objectives were to determine the phylogenetic position of Rhodotorula JG1b within the Rhodotorula genus and to identify

\footnotetext{
${ }^{1}$ Department of Natural Resource Sciences, McGill University, Sainte-Anne-de-Bellevue, QC, Canada. ${ }^{2}$ Department of Biology, Biotechnical Faculty, University of Ljubljana, Ljubljana, Slovenia. ${ }^{3}$ Lars Bolund Institute of Regenerative Medicine, BGI-Qingdao, Qingdao, China. ${ }^{4}$ Agricultural Institute of Slovenia, Ljubljana, Slovenia. ${ }^{5} \mathrm{NASA}$ Ames Research Center, Moffett Field, CA, USA. ${ }^{\circledR}$ email: Iyle.whyte@mcgill.ca
} 
and characterize its metabolic activity pathways and regulatory mechanisms in response to cold. To determine $R$. frigidialcoholis' response to colder temperatures, we characterized this strain's metabolic capabilities at $0{ }^{\circ} \mathrm{C}$, performed transcriptomic mRNAseq analysis in cultures grown at 0 and $23^{\circ} \mathrm{C}$, and assessed this species' ethanol production capability at $0^{\circ} \mathrm{C}$ compared to $23^{\circ} \mathrm{C}$. In addition, we analyzed the short non-coding RNAs to determine their role in post-transcriptional gene regulation to cold adaptation.

\section{MATERIALS AND METHODS Taxonomic analysis}

Rhodotorula JG1b was isolated from ice-cemented permafrost soil in University Valley (Antarctica) (24). To determine the phylogenetic position of Rhodotorula JG1b, we performed phylogenetic, phenotypic, metabolic, and physiological characterization of this strain. Phylogenetic characterization and analyses were performed using the small and large subunits of the rRNA gene, the internal transcribed spacers (ITS) 1 and 2, translation elongation factor 1-a (TEF), and cytochrome $b$ as well as publicly available whole genome sequences of the Rhodotorula genus (Supplementary Fig. S1). Detailed methodology of the phylogenetic analyses is described in the Supplementary material (Supplementary File 1 and Supplementary Fig. S2).

The morphology of the strain was determined by growth on potato dextrose agar (PDA), malt extract agar (MEA), cornmeal agar (CMA), and yeast extract peptone dextrose (YPD) [30], after incubation at $15^{\circ} \mathrm{C}$ for 14 days. Morphological characters of pure cultures were observed on MEA with Nomarski interference contrast optics on an Olympus BX-51 microscope, colony color was described according to [31]. Growth at different temperatures $\left(0,4,15,20,24,30\right.$ and $\left.37^{\circ} \mathrm{C}\right)$ was determined in microtiter plates in liquid medium yeast nitrogen base. Three inocula were used $(A, B, C)$ of optical density $0.4,0.2$, and 0.02 , respectively, with 8 replicates/inocula. The growth curves were determined via OD at $590 \mathrm{~nm}$. Assimilation of carbon and nitrogen sources were studied on three different sets of Phenotypic MicroArray (PM; Biolog) plates, PM1 MicroPlate and PM2A MicroPlate for carbon metabolism, and PM3B MicroPlate for nitrogen metabolism, as described by Viti et al., incubated at $20^{\circ} \mathrm{C}$ for up to 14 days. Fermentation of D-glucose was tested in liquid medium with a $2 \%$ solution of sugar with Bromothymol blue and Durham tube [30], after an incubation of 12 days at $5,15,20,30$, and $37^{\circ} \mathrm{C}$.

\section{Culturing and growth conditions}

For all subsequent experiments, Rhodotorula JG1b was cultured on PDB agar (HIMEDIA, Shenzhen, China). Biological triplicates of Rhodotorula JG1b were grown in $50 \mathrm{ml}$ liquid cultures to early exponential phase in PDB at $0^{\circ} \mathrm{C}$ without shaking, and at $23^{\circ} \mathrm{C}$ with shaking at $150 \mathrm{RPM}$. Growth was monitored using $\mathrm{OD}_{600}$ and converted to viable cell numbers $/ \mathrm{ml}$ based on CFU counts. Lack of shaking was necessary for the $0^{\circ} \mathrm{C}$ treatment to allow growth at this low temperature since growth was not observed under shaking conditions at $0^{\circ} \mathrm{C}$. We separately ensured that the $0^{\circ} \mathrm{C}$ nonshaking cultures remained aerobic $(11.5 \pm 0.98 \mathrm{mg} \mathrm{O} / /)$ during growth by monitoring $\mathrm{O}_{2}$ concentrations using a Piccolo $\mathrm{O}_{2}$ Fiber-Optic Oxygen Meter (Pyroscience, Aachen, Germany). $\mathrm{O}_{2}$ was not limited in this treatment, due to small culturing volume, slower growth rate at low temperature, and increase of oxygen solubility at lower temperature [32].

\section{Phenotypic MicroArray analysis}

To physiologically characterize Rhodotorula JG1b metabolic capabilities under cold conditions, its assimilation of different carbon and nitrogen sources at $0{ }^{\circ} \mathrm{C}$ was assessed using the Biolog Phenotypic MicroArray (PM) technology (Biolog, Hayward, CA, United States). PM1 MicroPlate and PM2A MicroPlate were used for determining carbon utilization, and PM3B MicroPlate was used for nitrogen utilization. The plates were inoculated following Viti et al. protocol [33] with the following modifications: the initial inoculum was adjusted to $\mathrm{OD}_{600} 0.200$, and the Biolog Dye Mix G was used. The duplicate plates were incubated at $0{ }^{\circ} \mathrm{C}$, and at room temperature of $23^{\circ} \mathrm{C}$ for comparison. Absorbance readings $(590 \mathrm{~nm})$ were taken at days $0,1,2,6,9,14,21,28,42,56,70$, and 91 of incubation on a SpectraMax M2e microplate reader (Molecular Devices, San Jose, CA, United States). The measurements were normalized by the subtraction of both the $T_{0}$ and the negative control. Absorbance values $>0.2$ were considered as positive values as previously described [34].

\section{RNA extraction, library preparation, and sequencing}

To perform transcriptomic analysis, biological triplicate Rhodotorula JG1b cultures of each condition were grown as described in section "Culturing and growth conditions" to early exponential phase: $\mathrm{OD}_{600} 0.95$ at $0{ }^{\circ} \mathrm{C}$; and $\mathrm{OD}_{600} 6.20$ at $23^{\circ} \mathrm{C}$. RNA was extracted using the Direct-zol RNA Miniprep Plus kit (\#R2070) from Zymo Research (Irvine, CA, United States) on cultures with an initial resuspended pellet of approximately $10^{8}$ cells following the manufacturer's instructions. An additional step of bead beating was also performed where resuspended cell suspensions were bead beat with $1: 3$ sterile glass beads $(0.1 \mathrm{~mm})$ for $45 \mathrm{~s}$ using the Mini-BeadBeater-24 (Biospec Products, Bartlesville, OK, United States) and centrifuged at 10,000 RCF for 2 min. Following a DNAse treatment with the TURBO DNAse kit (Invitrogen, Carlsbad, CA, United States), the RNA concentration was assessed with the Qubit 3.0 Fluorometer (Thermo Fisher Scientific, Waltham, MA, United States). RNA integrity was analyzed with the RNA Nano 6000 Assay Kit on the 2100 Bioanalyzer system (Agilent Technologies, Santa Clara, CA, United States). mRNA libraries were prepared following the TruSeq Stranded mRNA LT Sample Prep Kit (Illumina, San Diego, CA, United States) with an input material of $1.2 \mu \mathrm{g}$ of total RNA. Libraries band sizes were confirmed on a $4 \%$ agarose gel.

We prepared small RNA (sRNA) (>200 nt) libraries for sequencing since we noted higher prevalence of sRNAs in the $0^{\circ} \mathrm{C}$ cultures compared to the $23^{\circ} \mathrm{C}$ cultures (Supplementary Fig. S3). SRNA libraries were prepared following the NEBNext Multiplex Small RNALibrary Prep Workflow (New England Biolabs, Ipswich, MA, United States). The cDNA constructs were purified with the Monarch PCR and DNA kit from New England Biolabs. Libraries band sizes were assessed with the Agilent High Sensitivity DNA kit on the 2100 Bioanalyzer system. Library bands from 105 to $210 \mathrm{bp}$ were selected using the Pippin Prep 3\% Agarose Gel Cassette from Sage Science (Beverly, MA, United States). mRNA sequencing was performed by RNASeq on the MiSeq system (Illumina) using the Reagent Kit v2 50 cycles and a $1 \times 55$ bp single-end configuration. sRNA sequencing was performed by RNASeq on the MiSeq system (Illumina) using the Reagent Kit v2 300 cycles and a $2 \times 151$ bp paired-end configuration. mRNA and sRNA raw sequence files (fastq) were uploaded to GenBank with accession id: PRJNA631292

\section{Bioinformatics}

The mRNA raw sequencing reads were quasi-mapped and quantified with Salmon software [35] to the genome of Rhodotorula JG1b with the GenBank number GCA_001541205.1, and JGI IMG Genome ID 276120717 [24]. The analysis of the resulting quantifications was performed in $\mathrm{R}$ using the package DESeq2 [36]. Low count genes with less than 10 reads were removed before normalization of the samples. Hierarchical clustering and principal component analyses were used to check if the samples within the treatment (biological replicates) were more similar than between the treatments. The $p$ value threshold for differential expression was 0.05 . Log 2 fold-change threshold was not used, but $\log _{2}$ fold-change shrinking was used for data visualization and gene ranking. Genes with a $\log _{2}$ foldchange $\left(\log _{2} \mathrm{FC}\right)$ ratio $\geq|1.5|$, and a $p$ value $<0.05$ were considered as differentially expressed between the two growth conditions. Predicted protein KEGG assignments for each differentially expressed gene were downloaded from BlastKOALA website [37], to obtain function and metabolic pathway assignments. Heatmaps of the relative abundance of transcript expression of specific pathways were created. For those heatmaps, relative gene abundances of each sample were calculated by taking gene abundance (corrected on sequencing depth and length) and subtracting the average abundance across all samples.

sRNA raw sequence data were trimmed for adapter sequences and quantified using the CLC Genomics Workbench version 12 (https://www. qiagenbioinformatics.com/). The short rRNA fragments ( $\mathrm{rRF}$ )/microRNAs (miRNAs) sequences (15-30 nt) were extracted and counted from the total sRNA sequences using the CLC Genomics Workbench Small RNA Analysis tool. Identical short $\mathrm{rRF} / \mathrm{miRNAs}$ were grouped together and low count sequences with less than 50 reads were removed. The short rRFs/miRNAs were normalized based on sequencing coverage and to the ratio of sRNA/ Total RNA, and differential expression was analyzed, with a $p$ value threshold of 0.05 . PCoA analysis (Bray-Curtis distance) was performed with the CLC Genomics Workbench version 12. The genomic origin of the ten highest expressed short rRFs/miRNAs were predicted with BLAST [38] by comparing to Rhodotorula genomes, and their potential mRNA targets were predicted using the RNAhybrid algorithm with the short rRFs/miRNAs sequences and all possible transcribed mRNA sequences as potential targets $[39,40]$. The predicted protein mRNA targets of the short rRFs/ miRNAs were identified with KEGG [37]. 


\section{Ethanol production assay}

To corroborate the phenotypic and transcriptomic results, Rhodotorula frigidialcoholis' ethanol production capability was assessed. Triplicate Rhodotorula JG1b cultures were grown in identical media and conditions as with transcriptomic analysis, detailed in section "Culturing and growth conditions." $\mathrm{OD}_{600}$ of the cultures was measured at regular time points for 83 days at $0{ }^{\circ} \mathrm{C}$ and for $17 \mathrm{~h}$ at $23^{\circ} \mathrm{C}$. Aliquots $(1 \mathrm{ml})$ of the cultures at each time point were analyzed for ethanol production using the EnzyChrom Ethanol Assay Kit (BioAssay System, Hayward, CA, United States). Analysis of the ethanol production was done by comparing the trendline of ethanol concentration to the trendline of cell density over time. Ethanol concentrations were normalized to the ethanol evaporation rate that was experimentally determined using sterile PDB broth inoculated with $0.05 \%$ of ethanol.

\section{RESULTS AND DISCUSSION \\ Taxonomy and description of Rhodotorula frigidialcoholis sp. nov}

The Rhodotorula yeast strain JG1b isolated from the upperelevation McMurdo Dry Valleys of Antarctica [24] was determined to be a novel species based on phylogenetic and phenotypic analyses. Bayesian analysis of aligned, concatenated 18S, ITS, D1/ D2 domains of $28 \mathrm{~S}$ rRNA, and TEF sequences of species within the genus Rhodotorula resulted in a consensus tree shown in Supplementary Fig. S1A, while the phylogenetic tree based on available complete Rhodotorula genomes is shown in Supplementary Fig. S1B. According to these analyses, the JG1b strain forms a strongly supported monophyletic group within Rhodotorula species and is highly supported as the sister clade of $R$. mucilaginosa (bootstrap $=100 \%$ ). Clustering analysis of the data obtained from 190 carbon and 95 nitrogen assimilation assays confirmed that Rhodotorula JG1b is distinct from other Rhodotorula species included in the Biolog phenotyping experiment (Supplementary Fig. S1C). Contrary to $R$. mucilaginosa and $R$. alborubescens, Rhodotorula JG1b is able to assimilate L-rhamnose, dulcitol, L-sorbose, and is unable to assimilate citrate and Dcellobiose (Supplementary Table S1). Based on the results presented here, we strongly suggest that Rhodotorula sp. JG1b is a novel species in the Rhodotorula genus. Accordingly, the species is taxonomically introduced herewith as Rhodotorula frigidialcoholis D. Touchette \& P. Zalar, sp. nov. (frigidus in Latin meaning cold, alcohilis in Latin genitive case of alcohol; of cold alcohol, referring to ability of alcohol production in cold). Morphological characters of $R$. frigidialcoholis are shown in Fig. 1. Holotype for Rhodotorula frigidialcoholisdesignated herewith: EXF-10854H, permanently preserved and metabolically inactive deposit of the Culture Collection of Extremophilic Fungi Ex (EXF), University of Ljubljana, Slovenia. Ex-type cultures: EXF10854 and CBS 16468 (Westerdijk Fungal Biodiversity Institute, Utrecht, The Netherlands). The strain EXF-10854 was isolated as JG1b from 150,000-year-old ice-cemented permafrost of University Valley, the upper-elevation McMurdo Dry Valleys of Antarctica in 2009 by Jacqueline Goordial (24). DNA sequence accession numbers derived from type: MT569975 (18S rRNA), MT569976 (D1/D2 of 28S rRNA), MT560678 (ITS), MT584855 (TEF1). MycoBank accession number for Rhodotorula frigidialcoholis is MB 835866. Diagnosis: After a 14-day incubation at $15^{\circ} \mathrm{C}$ on $5 \%$ MEA agar, the streaked cultures are slimy, liquid and shinning with entire margin, greyish red (7B6) (Fig. 1). The cells are subglobose, $3.5 \times 4.5 \mu \mathrm{m}$, and encapsulated, budding is unipolar (Fig. 1). Fermentation was not observed. Assimilated compounds are Dglucose, sucrose, raffinose, galactose, trehalose, maltose, melezitose, L-sorbose, L-rhamnose, D-xylose, Dl-arabinose, D-ribose, glycerol, galactitol, D-mannitol, and xylitol. Not observed assimilated compounds are inulin, melibiose, lactose, methyl A-Dglucoside, cellobiose, erythritol, myo-inositol, citrate, D-glucosamine, N-acetyl-D-glucosamine, nitrate, and nitrite. Hydrolysis of urea is positive. A complete list of assimilated and not assimilated compounds is listed in the Supplementary Table S1. Growth from 0 to $37^{\circ} \mathrm{C}$ (Supplementary Fig. S4). Strain JG1b was previously described as able to grow as low as $-10^{\circ} \mathrm{C}[1,24]$, but appears to have lost subzero growth capabilities through sub-culturing and long-term storage at $-80^{\circ} \mathrm{C}$, as in this study $R$. frigidialcoholis was only successfully grown at $0^{\circ} \mathrm{C}$ while incubated in a PDB $50 \mathrm{ml}$
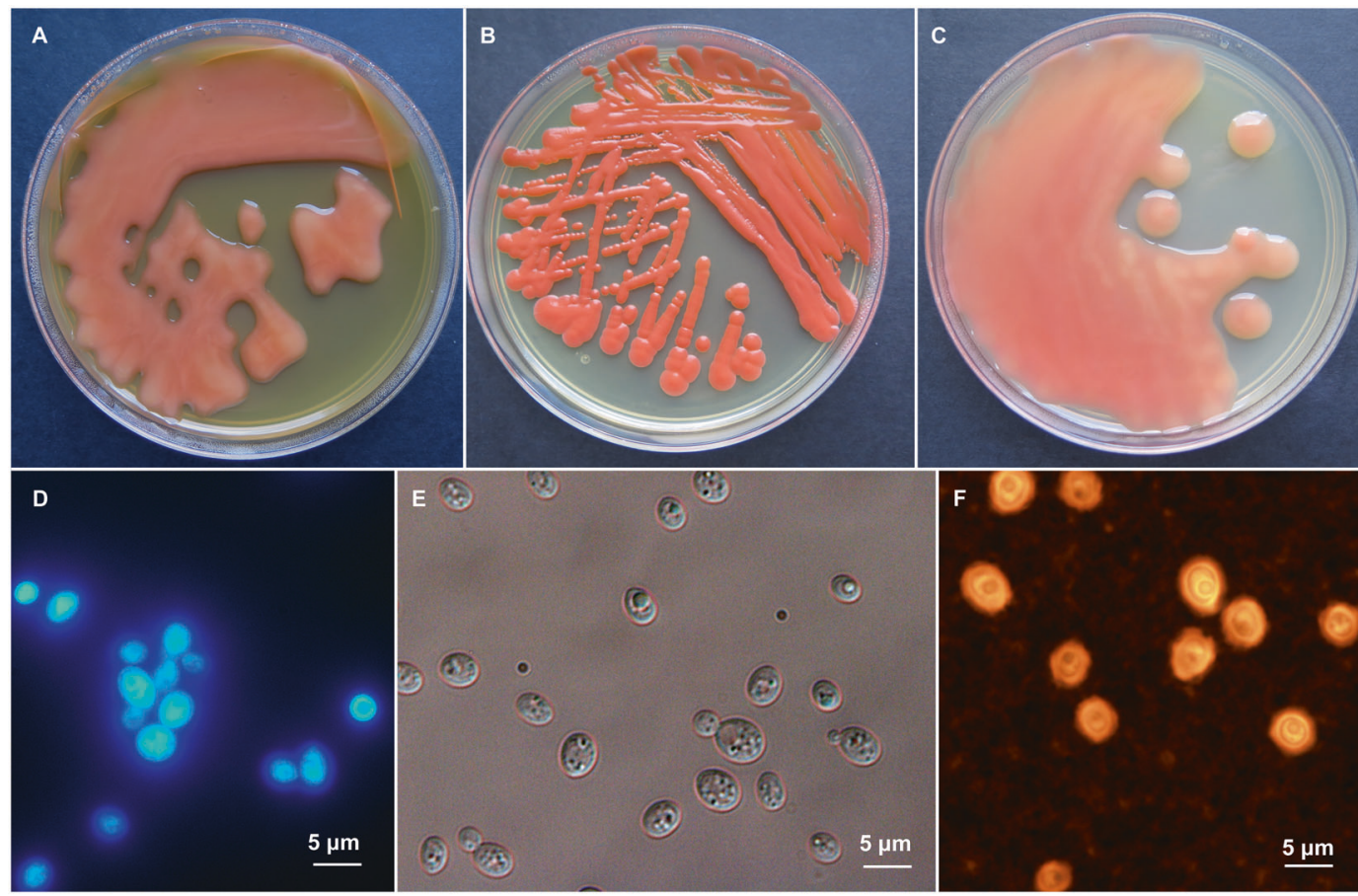

Fig. 1 Morphology of Rhodotorula frigidialcoholis. A-C Cultures of R. frigidialcoholis on PDA (A), YPD (B), and MEA (C) in $9 \mathrm{~cm}$ Petri dishes after 2 weeks of incubation at $15^{\circ} \mathrm{C}$. Micromorphology of cells stained with calcofluor white viewed with fluorescent microscopy (D), mounted in water, viewed by DIC (E), stained with India ink, viewed with light microscopy (F), all after 2 weeks of growth at $15^{\circ} \mathrm{C}$. 


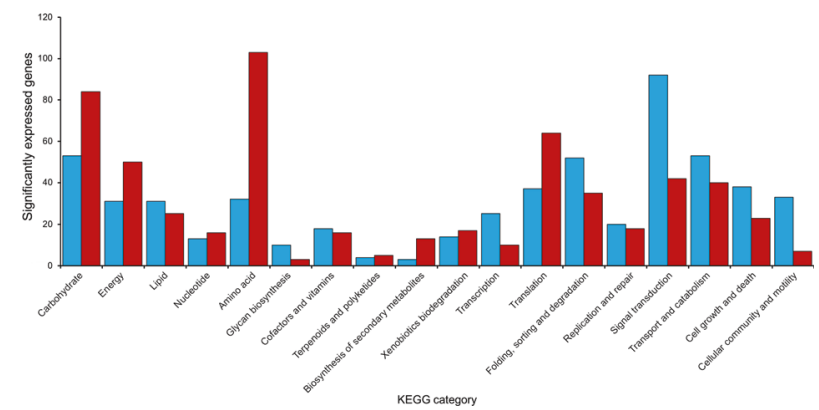

Fig. 2 Number of gene significantly differentially expressed for each KEGG metabolic pathways and cellular processes in Rhodotorula frigidialcoholis at $0^{\circ} \mathrm{C}$ compared to $23^{\circ} \mathrm{C}$. The total number of genes in each KEGG category that showed significant ( $p$ $<0.05)$ upregulation $\left(\geq 1.5 \log _{2} \mathrm{FC}\right)$ at $0{ }^{\circ} \mathrm{C}$ are indicated in blue. The total number of genes in each KEGG category that showed significant $(p<0.05)$ upregulation $\left(\geq 1.5 \quad \log _{2} \mathrm{FC}\right)$ at $23^{\circ} \mathrm{C}$ are indicated in red. Blue and red are equivalent to light gray and dark gray in the printed version.

liquid culture, without shaking. An extended description of Rhodotorula frigidialcoholis is detailed in the Supplementary material (Supplementary file 1) a complete list of assimilated and not assimilated compounds in the Table S1.

\section{Phenotypic MicroArray response at cold temperatures}

To characterize $R$. frigidialcoholis metabolic capabilities at low temperature, its substrate utilization was assessed through the Biolog Phenotypic MicroArray that determines the ability of a microorganism to utilize a variety of sole carbon or nitrogen sources [41]. Positive detection of metabolic activity relies on the transfer of electrons from the growth substrate, through the electron transport chain (ETC), to the tetrazolium redox dye, transforming the dye into a violet colored reduced form [41, 42]. After 91 days of incubation, $R$. frigidialcoholis utilized 35 on 190 substrates as a sole carbon source at $0^{\circ} \mathrm{C}$ for growth (Supplementary Table S2). Only two (L-Proline, L-Pyroglutamate) of the 95 nitrogen sources were utilized as sole nitrogen sources at $0{ }^{\circ} \mathrm{C}$ after 91 days of incubation (Supplementary Table S2). Surprisingly, of the 35 positive carbon substrates, only six (DTrehalose, D,L-a-Glycerol Phosphate, D-Mannose, Tween 20, Tween 40 , and Tween 80 ) showed reduction of the dye and parallel evidence of microbial growth (Supplementary Table S2). This was puzzling because in the remaining 29 carbon sources, we observed active growth in the wells (based on $\mathrm{OD}_{600}$ and visual inspection) but without reduction of the dye; this growth was confirmed by streak plates. As the tetrazolium dye is reduced via the ETC [43], the observation of growth but no reduction of the dye suggest that at $0{ }^{\circ} \mathrm{C} R$. frigidialcoholis potentially produces most of the energy for cellular growth through metabolic pathways other than ETC, such as glycolysis or perhaps fermentation. Damage to the mitochondrial DNA and the ETC in $S$. cerevisiae was previously shown to decrease tetrazolium dye reduction [44] while yeast mitochondria mutants have respiratory dysfunction and an inability to reduce tetrazolium salts [45-47]. Therefore, growth of $R$. frigidialcoholis coupled with a lack of tetrazolium dye reduction suggests that cell respiration through the mitochondria and ETC was significantly reduced while growth was still maintained at $0{ }^{\circ} \mathrm{C}$.

\section{mRNA transcriptional responses to cold temperature}

To determine $R$. frigidialcoholis transcriptional response to cold, we performed comparative mRNA transcriptomic analysis on triplicate exponential phase cultures of $R$. frigidialcoholis grown at 0 and $23^{\circ} \mathrm{C}$. Overall, 1772 genes were significantly differentially expressed $(p<0.05)$ between these two conditions, including

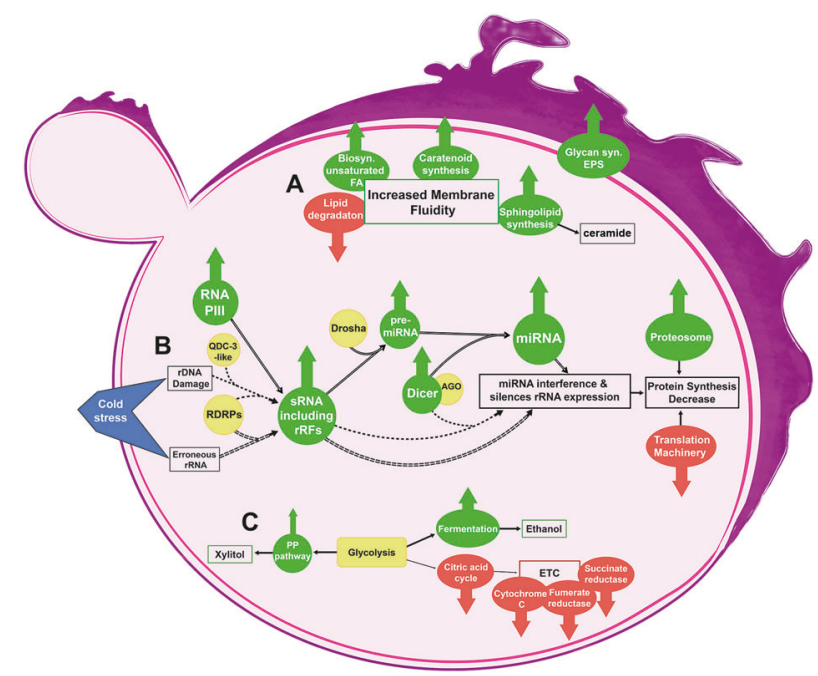

Fig. 3 Diagram showing major processes and pathways increases during growth at $0^{\circ} \mathrm{C}$ in Rhodotorula frigidialcoholis. A Cold induced modifications to $R$. frigidialcoholis membrane. B Potential cold induced RNAi mechanisms in $R$. frigidialcoholis. Hollow arrows represent the miRNA and short rRFs (miRNA-like) mechanisms $[105,107]$, dashed arrows represent the rRFs derived from rDNA damage mechanism [115], double-dashed arrows represent the rFRs derived from erroneous rRNA mechanism resulting in rRNA synthesis inhibition [114]. C Cold induced switch in $R$. frigidialcoholis metabolism. A list of the abbreviations is included in the Supplementary material (Supplementary Table S5).

994 genes overexpressed, and 778 genes downregulated at $0{ }^{\circ} \mathrm{C}$ compared to $23^{\circ} \mathrm{C}$ (Supplementary Tables S3 and S4). Of these significantly differentially expressed genes, $52 \%$ were annotated with KEGG. The major global differences in transcript abundance between 0 and $23^{\circ} \mathrm{C}$ were found in genes related to carbohydrate metabolism, energy metabolism, lipid metabolism and signal transduction, glycan biosynthesis/metabolism, transcription machinery, translation, and amino acid metabolism (Fig. 2). While protein levels are not always correlated with the transcript levels as they are also influenced by post-transcriptional, translational, and degradation regulation, mRNA levels do partially correlate with protein abundance [48] and are informative to deduce overall metabolic processes [49].

Cellular membrane and signal transduction. Maintenance in membrane fluidity through lipid membrane component modification is one of the best-known adaption strategies in microorganisms living in cold environments [23]. Overall, $R$. frigidialcoholis overexpressed genes involved in lipid metabolism at $0^{\circ} \mathrm{C}$ compared to $23^{\circ} \mathrm{C}$ (Figs 2 and 3 and Supplementary Table S5). Similar to previous studies, genes related to unsaturated fattyacids (FA) biosynthesis were overall overexpressed at $0^{\circ} \mathrm{C}$ indicating increased unsaturated FA concentrations maintain membrane fluidity at cold temperatures [50-53]. Relatively high amounts of unsaturated FAs were also reported in other psychrophilic basidiomycetous yeasts isolated from Antarctic and Patagonian ecosystems [54-56].

Sphingolipid metabolism genes were also highly overexpressed at $0{ }^{\circ} \mathrm{C}$ compared to $23^{\circ} \mathrm{C}$ in $R$. frigidialcoholis (Supplementary Fig. S4) presumably leading to increased biosynthesis of ceramide and phytoceramide. Phytoceramide synthesis genes were previously shown to be consistently up-regulated during low temperatures fermentation $\left(12^{\circ} \mathrm{C}\right)$ in Saccharomyces cerevisiae $[57,58]$. Ceramide and other sphingolipid products can induce cell cycle arrest [59] indicating growth suppression in $R$. frigidialcoholis may be a response to freezing conditions in Antarctic permafrost habitats. 
Multiple Rhodotorula species produce lipid antioxidants and photoprotective carotenoids [60-62], especially high concentrations of $\beta$-carotene, torulene, and torularhodin. Carotenoids help maintain membrane rigidity in cold-adapted microorganisms as they balance out the higher percentage of unsaturated FAs $[50,63]$. At $0{ }^{\circ} \mathrm{C}, R$. frigidialcoholis overexpressed the phytoene desaturase gene $(\mathrm{FC}=$ 4.46, $p=0.00$ ) involved in the production of torulene and $\beta$ carotene precursors. This is corroborated by our observation of a darker pink color in $R$. frigidialcoholis grown at $0{ }^{\circ} \mathrm{C}$ compared to $23^{\circ} \mathrm{C}$. Carotenoids play an important role in UV and sunlight protection in yeast $[61,64,65]$. Thus, at $0^{\circ} \mathrm{C}, R$. frigidialcoholis may increase carotenoid biosynthesis both to regulate membrane fluidity and as an adaption to solar irradiation or long-term background $y$ radiation in the extreme University Valley permafrost environment.

The PI3k-Akt, Hippo, TGFß, VEGF, RAS, MAPK, RAP1, FoxO, and Wnt cell signal transduction pathways genes were overexpressed at $0{ }^{\circ} \mathrm{C}$ (Fig. 2 and Supplementary Table S4); these pathways are related to gene regulation, actin cytoskeleton formation, and cellular adhesion, and are activated by transmembrane receptors. While little is known about cold response signal transduction modulation, it has been linked to freezing temperature survival in plants $[66,67]$. Perception of cold in Rhodotorula by cold-sensitive membrane receptors may also lead to a cascade response, thus preparing it for cold temperatures. In yeasts, mitogen activated protein kinase cascades play major roles in gene transcription and actin cytoskeletal organization [68]. Thus, $R$. frigidialcoholis may overexpress genes coding for the formation of the actin cytoskeleton (cdc42, actin $\delta / \gamma$ subunits) at $0^{\circ} \mathrm{C}$ (Supplementary Table S4) as a way to transport lipids (biosynthesis of which is also overexpressed at $0{ }^{\circ} \mathrm{C}$ ) to the membrane and maintain membrane fluidity (Figs 2 and 3 and Supplementary Fig. S4).

Differential expression of homologous proteins between 0 and $23^{\circ} \mathrm{C}$. We observed 19 differentially expressed homologous protein categories between $R$. frigidialcoholis cultures at 0 and $23^{\circ} \mathrm{C}$ (Supplementary Table S6). Eight of these differentially expressed homologous protein categories were related to membrane structure and transport across membranes. For
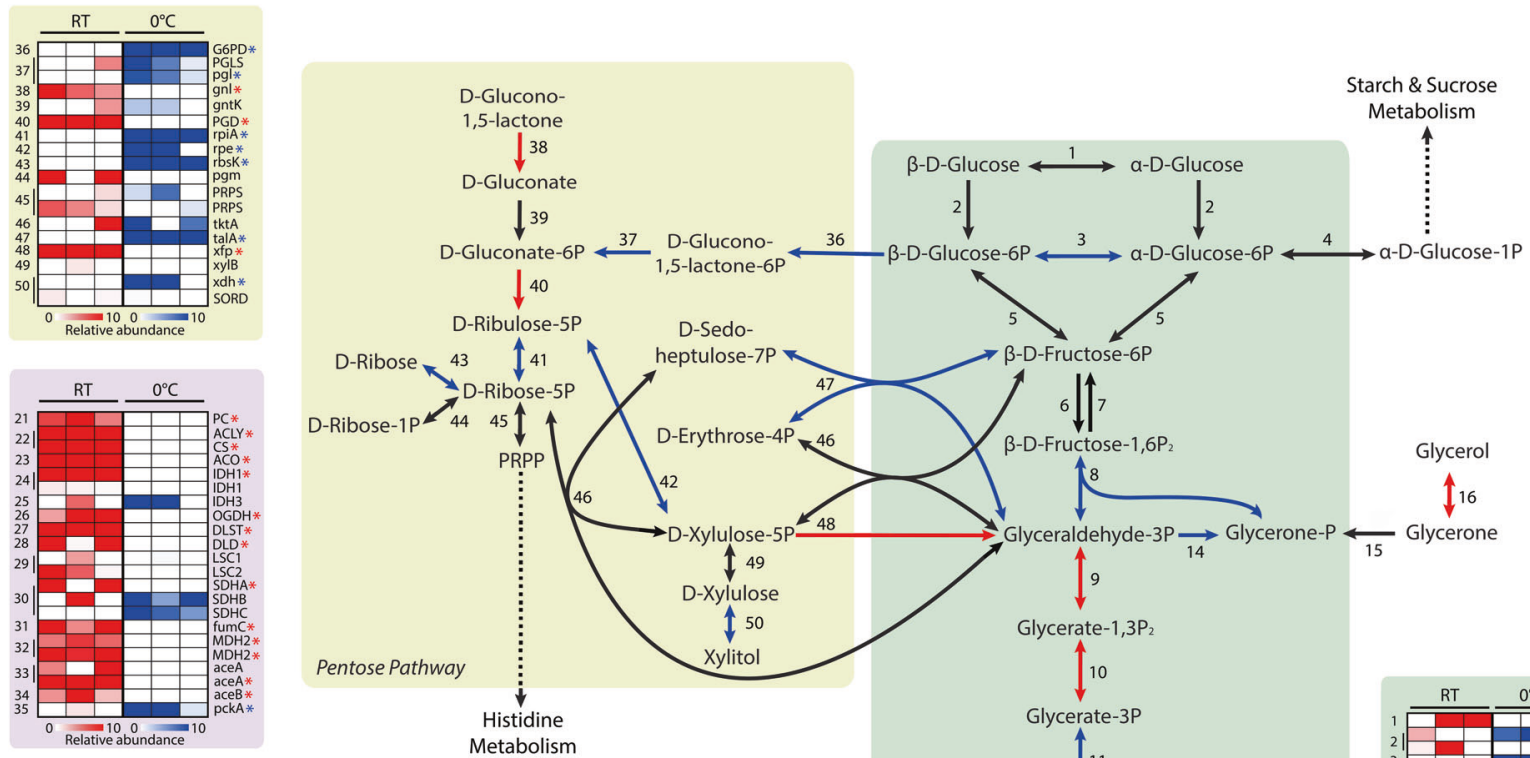

Pentose Pathwa
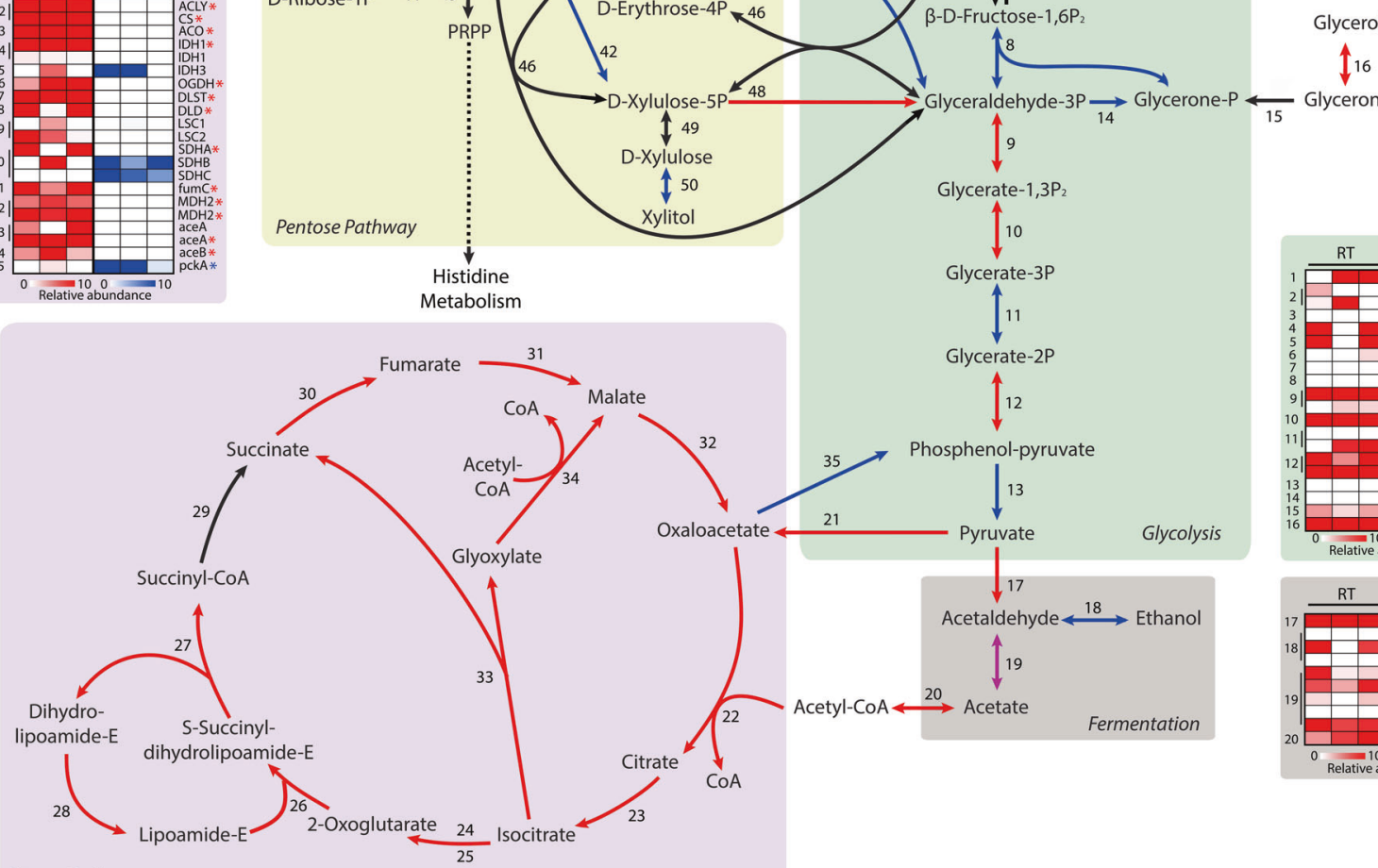

Citrate Cycle

Fig. 4 Reconstruction of the Rhodotorula frigidialcoholis major carbohydrate metabolic pathways mapped with transcriptomic data. Reconstructed carbohydrate metabolic pathways of $R$. frigidialcoholis based on the KEGG gene annotations and their relative differential gene expression profiles of each triplicate culture. The genes with a significant $(p<0.05)$ differential expression of $\geq 1.5 \log _{2} \mathrm{FC}$ are indicated with an arrow (pathway) and a star (heatmap) in blue (overexpressed at $0^{\circ} \mathrm{C}$ ), red (overexpression at $23^{\circ} \mathrm{C}$ ), or purple (different homolog overexpressed at both 0 and $23^{\circ} \mathrm{C}$ ). The numbers in the pathways correspond to the numbers in the heatmaps. For the heatmaps, blue indicates an overexpression of the gene at $0^{\circ} \mathrm{C}$, red indicates and overexpression of the gene at $23^{\circ} \mathrm{C}$, and white indicates genes that are not differentially expressed between the two temperatures. A list of the abbreviations is included in the Supplementary material (Supplementary Table S5). . 
example, YAT, an amino acid transporter, had three homolog genes overexpressed at $0{ }^{\circ} \mathrm{C}$, two homologs overexpressed at $23^{\circ} \mathrm{C}$, and two homologs that were not differentially expressed between the two temperatures (Supplementary Table S6). Homologous proteins arise from gene duplication and sometimes gain new functions [69]. Gene duplication, genome redundancy, and paralogous (homologs) genes increase the organism's ability to grow under varied conditions (e.g., temperatures, substrates, $\mathrm{pH}$, salinity) $[2,70,71]$.

Glycan biosynthesis and metabolism. A significant increase in glycan biosynthesis genes was observed in $R$. frigidialcoholis at $0^{\circ}$ C compared to $23^{\circ} \mathrm{C}$ (Figs 2 and 3). Glycans are carbohydratebased polymers associated with cellular protection and storage [72] and are also important component of glycoproteins, including cell-surface membrane proteins, such as receptors and adhesion proteins [72, 73]. Rhodotorula species produce mannan, a mannose glycan extracellular polysaccharide (EPS) [74, 75]. Microbial EPS are components of microbial biofilms and constitute a protective matrix against the desiccation and environmental fluctuations [76, 77], including freeze-thaw damage [78]. At $0^{\circ} \mathrm{C}$, $R$. frigidialcoholis overexpressed genes involved in GDP-Dmannose synthesis (a glycan biosynthesis precursor), and overexpressed multiple genes coding for glycosyltransferase and glycosidases (Supplementary Table S4), which regulate glycans [79]. These results indicate that $R$. frigidialcoholis increases EPS synthesis at $0{ }^{\circ} \mathrm{C}$ through overexpression of mannan and other glycoproteins as adaptation to desiccation and freeze-thaw cycles of the Antarctic University Valley permafrost environment.

Transcription, translation, and amino acid metabolism. R. frigidialcoholis overexpressed multiple genes involved in the transcriptional machinery at $0{ }^{\circ} \mathrm{C}$ compared to $23^{\circ} \mathrm{C}$ (Figs 2 and 3), including the overexpression of genes coding for the three RNA polymerase complexes (RNA Polymerase I, II, and III and the RNA polymerase II transcription factor) (Supplementary Table S4). A similar increase in the transcriptional machinery at 10 and $4{ }^{\circ} \mathrm{C}$ was reported in other yeasts [80-83]. Contrariwise, $R$. frigidialcoholis decreased expression of genes related to translation at $0{ }^{\circ} \mathrm{C}$ compared to $23^{\circ} \mathrm{C}$, such as the downregulation of genes involved in ribosomal protein synthesis and aminoacyl-tRNAs. In addition, $R$. frigidialcoholis overexpressed proteasome genes involved in protein degradation at $0{ }^{\circ} \mathrm{C}$. Cold temperatures can reduce translation efficiency and protein synthesis by inducing formation of secondary structures in DNA/RNA molecules and by inactivating ribosomes [4, 83, 84]. While amino acid biosynthesis genes were overall downregulated at $0^{\circ} \mathrm{C}$ compared to $23^{\circ} \mathrm{C}$ (Fig. 2), the bacterium Polaromonas sp. also downregulated genes involved in amino acid biosynthesis and transport [8]. Thus, at low temperatures, $R$. frigidialcoholis may decrease amino acid synthesis and transport to slow down translation and focus energy on cold acclimation, in parallel with increased protein degradation to possibly recycle amino acids. A notable exception was of upregulation of the histidine pathway; this could be due to its role in membrane cold sensor histidine kinases [85] as was reported in the basidiomycetous yeast Mrakia blollopis, which accumulated aromatic amino acids, such as histidine, at $-3^{\circ} \mathrm{C}$ compared to $10^{\circ} \mathrm{C}[86]$.

Carbohydrate metabolism and energy metabolism. The majority of carbohydrate metabolism expression differences between 0 and $23^{\circ} \mathrm{C}$ were in genes of the citrate cycle, pentose phosphate pathway (PPP), and alcohol fermentation. At $23^{\circ} \mathrm{C}, R$. frigidialcoholis significantly overexpressed citrate cycle genes as well as glycolysis genes linked to oxaloacetate and acetyl-CoA syntheses which feed into the citrate cycle (Fig. 4 and Supplementary

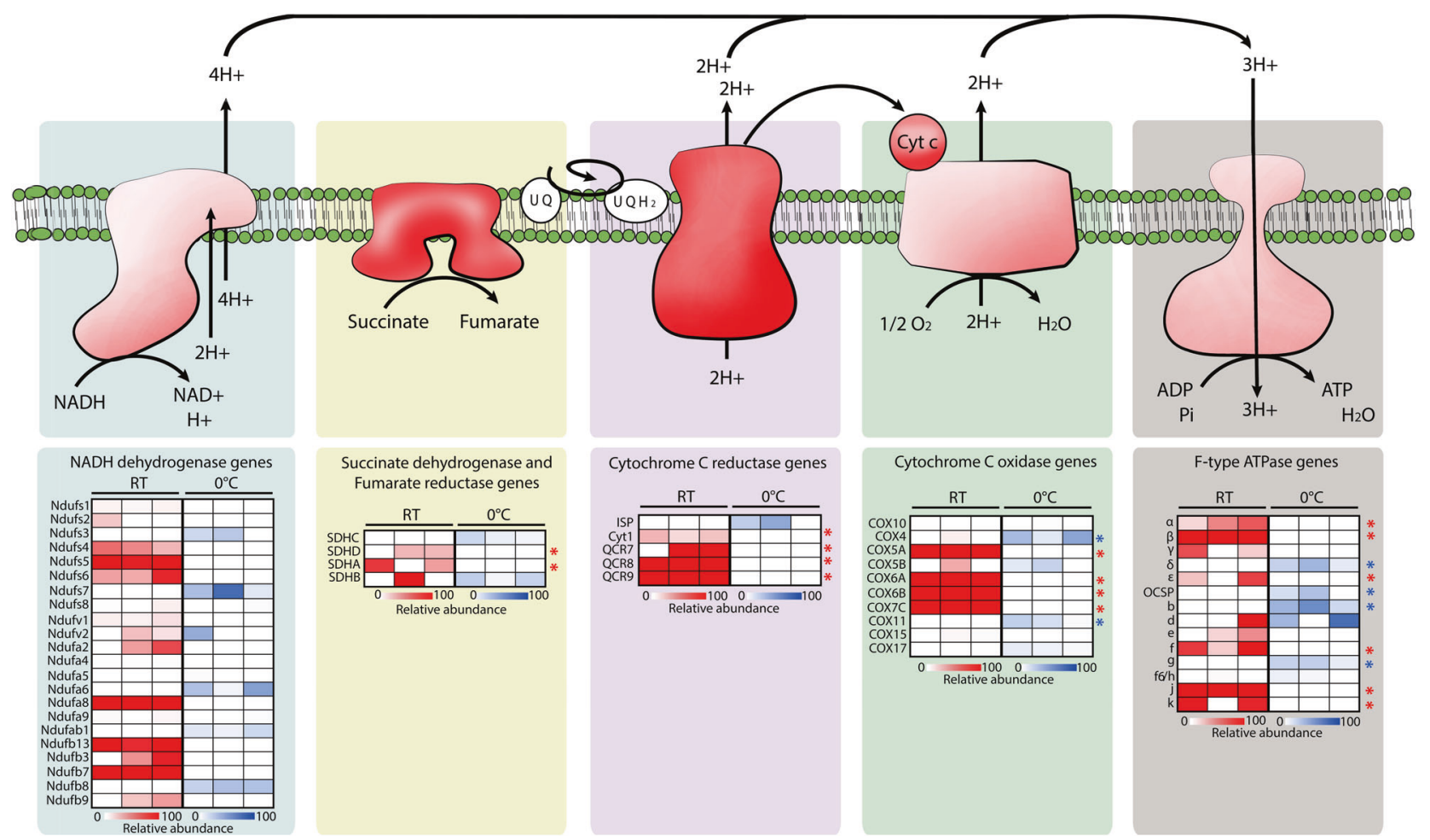

Fig. 5 Reconstruction of the Rhodotorula frigidialcoholis electron transport chain. Reconstructed electron transport chain (ETC) complexes of $R$. frigidialcoholis based on the KEGG genes annotation (top) and their relative differential gene expression profiles of the ETC subunits (bottom) of each triplicate culture. The genes with a significant $(p<0.05)$ differential expression of $\geq 1.5 \log _{2} \mathrm{FC}$ are indicated with a star in blue (overexpressed at $0{ }^{\circ} \mathrm{C}$ ), or red (overexpression at $23^{\circ} \mathrm{C}$ ). For the heatmaps, blue indicates an overexpression of the gene at $0{ }^{\circ} \mathrm{C}$, red indicates and overexpression of the gene at $23^{\circ} \mathrm{C}$, and white indicates genes that are not differentially expressed between the two temperatures. 
Table S5). In contrast, at $0{ }^{\circ} \mathrm{C}$, there was a significant increase in the expression of glycolysis genes feeding into ethanol production via fermentation and a significant increase in expression of the PPP genes (Fig. 4).

At $0{ }^{\circ} \mathrm{C}, R$. frigidialcoholis overexpressed genes coding for xylulose reductase $(F C=2.40, p=0.00)$, non-oxidative PPP enzymes, and alcohol dehydrogenase $(F C=8.05, p=0.00)$, while it downregulated gene coding for 6-phosphogluconate dehydrogenase ( $\mathrm{FC}=2.21, p=0.00$ ) (Fig. 4). The increase in PPP promotes the production of erythrose-4-phospate, allowing energy (NADH) conservation due to a reduction of the citrate cycle activity [87]. In yeast, including Rhodotorula, the PPP is involved in xylulose fermentation [88-90], while downregulation of 6phosphogluconate dehydrogenase activity is correlated with increased production of ethanol via xylulose fermentation [91]. Xylulose is fermented by xylulose reductase [88] to xylitol, a cryoprotectant and freezing point depressant in yeast [92]. The non-oxidative PPP enzymes can also increase the yield of ethanol fermentation through the conversion of pentose phosphate into intermediates of the glycolysis pathway [90]. A similar upregulation of alcohol dehydrogenase was also observed in the Arctic permafrost bacterium, Planococcus halocryophilus, grown at $-15^{\circ} \mathrm{C}$ [2]. These transcriptional changes in $R$. frigidialcoholis suggest that it adapts to cold temperature by increasing xylitol production and redirecting PPP molecules to ethanol fermentation, which would be beneficial for its survival in extreme cryoenvironments such as Antarctic University Valley.

Antioxidant production through proline-linked PPP was proposed in yeast [93] as an oxidative stress response to cold [87]. $R$. frigoalchoholis can use L-Proline as a sole carbon and nitrogen source (Supplementary Table S2), without reducing the tetrazolium redox dye, suggesting that L-Proline is used by Rhodotorula in the proline-linked PPP, leading to decreased citrate cycle activity. This may result in a decrease of the ETC activity and, consequently, the observed lack of tetrazolium dye reduction and a possible redirection of erythrose-4-phosphate to the fermentation. Overall, increased transcription of the PPP genes at cold temperatures could help $R$. frigidialcoholis to conserve NADH conservation, limit carbon loss through $\mathrm{CO}_{2}$, and enhance ethanol production to lower the freezing point.

Overall, a higher number of genes related to energy metabolism were downregulated at $0^{\circ} \mathrm{C}$ compared to $23^{\circ} \mathrm{C}$ (Fig. 2). In accordance with our carbohydrate metabolism results, this significant change was related to an overall downregulation of the genes encoding for the ETC subunits at $0{ }^{\circ} \mathrm{C}$ (Fig. 5). The temperature effect on the ETC activity remains unclear, but some studies confirm a general trend in decrease of ETC expression at lower temperatures in microorganisms [8, 94]. The University Valley permafrost bacterium, Rhodococcus JG3, also downregulated multiple ETC cytochromes when grown at $-5{ }^{\circ} \mathrm{C}$ compared to $23^{\circ} \mathrm{C}$ [8], and Pseudomonas putida downregulated its NADH and ETC succinate dehydrogenase at $10^{\circ} \mathrm{C}$ compared to $30^{\circ} \mathrm{C}$ [94]. The ETC gene expression patterns in $R$. frigidialcoholis supports our hypothesis related to the lack of dye reduction in the PM assay at $0{ }^{\circ} \mathrm{C}$ as being due to the downregulation of the ETC and a switch to fermentative metabolisms to produce energy. This is further supported by the observed switch in gene expression from the citrate cycle pathway at $23^{\circ} \mathrm{C}$ to the fermentation ethanol and xylitol pathways at $0{ }^{\circ} \mathrm{C}$ for primary energy production (Fig. 4).

\section{Ethanol production in Rhodotorula frigidialcoholis}

$R$. frigidialcoholis' switch in metabolism from mainly respiratory at $23^{\circ} \mathrm{C}$ to fermentative at $0{ }^{\circ} \mathrm{C}$ was experimentally confirmed as ethanol production was observed at $0^{\circ} \mathrm{C}$, at an average rate of $1.51 \times 10^{-5}$ moles of ethanol, per liter, per day (Fig. 6A), while ethanol production was not detected at $23^{\circ} \mathrm{C}$ (Fig. 6B). Other Rhodotorula species, R. minuta, R. mucilaginosa, and R. pallida, are capable of ethanol fermentation at $28^{\circ} \mathrm{C}$ [95]; however, ethanol
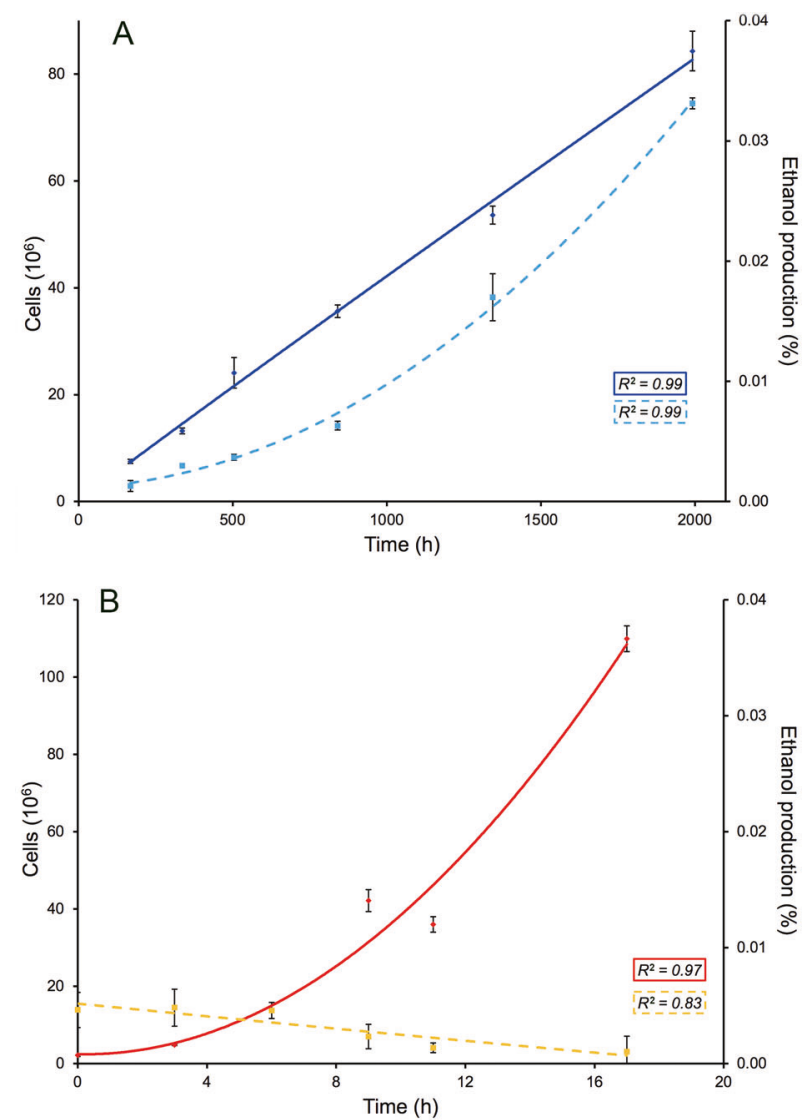

Fig. 6 Ethanol production and growth by Rhodotorula frigidialcoholis at 0 and $23^{\circ} \mathrm{C}$, in PDB media. A Cell growth (diamond, full line) and ethanol production (square, dashed lines) over time at $0^{\circ} \mathrm{C}$. B Cell growth (diamond, full line) and ethanol production (square, dashed lines) over time at $23^{\circ} \mathrm{C} . R^{2}$ values represent the coefficient of determination of the best fit polynomial trendline.

fermentation at low temperatures in Rhodotorula has not been reported. Yeast ethanol production at cold temperatures was reported with Saccharomyces cerevisiae in wine $\left(0\right.$ and $\left.2{ }^{\circ} \mathrm{C}\right)$ and beer $\left(6^{\circ} \mathrm{C}\right)$ production, although in these studies, fermentation was facilitated by an addition of biocatalysts [96-98]. To our knowledge, $R$. frigidialcoholis is able to naturally produce ethanol at the lowest recorded temperature for microbial fermentation. $R$. frigidialcoholis could then be studied on its potential to isolate cold-temperature active enzymes that promote cellulosic biomass decomposition through fermentation [99], favorizing biofuel production with less energy. Ethanol fermentation has also previously been linked with an increased production of carotenoids in yeast [100], which is consistent with $R$. frigidialcoholis transcriptomic results of increased carotenoid gene expression.

Combined together, downregulation of citrate cycle and ETC genes (Figs 4 and 5), overexpression of fermentation genes (Fig. 4), growth without reduction of the tetrazolium redox dye (Supplementary Table S2), and ethanol production at $0{ }^{\circ} \mathrm{C}$ but not at $23^{\circ} \mathrm{C}$ suggest that $R$. frigidialcoholis switches from a mainly respiratory metabolism when incubated at $23^{\circ} \mathrm{C}$ to a mainly fermentative metabolism when cultured in conditions similar to its natural environment at $0{ }^{\circ} \mathrm{C}$. Ethanol production in $R$. frigidialcoholis may be an ecologically significant adaptive response to subzero temperatures by both acting as a freezing point depressant reducing intracellular freezing and/or to decrease its environment freezing point enhancing the formation of subzero liquid brine vein microhabitats thought to exist within permafrost [101]. Ethanol production could also promote NADH conservation 

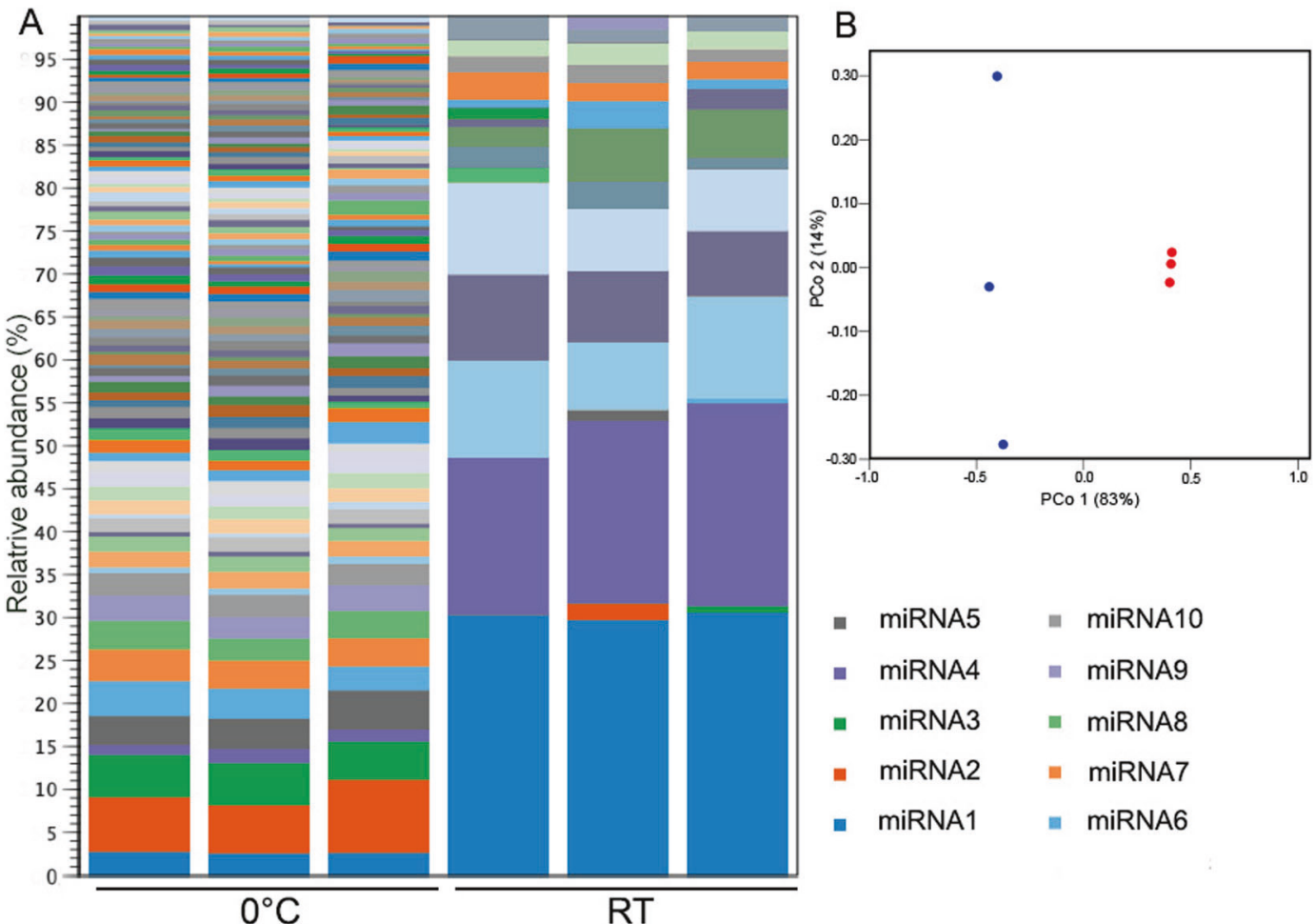

$$
\begin{aligned}
& \text { - miRNA5 }=\operatorname{miRNA10} \\
& \text { - miRNA4 } \\
& \text { - miRNA3 }=\text { miRNA8 } \\
& \text { - miRNA2 }=\text { miRNA7 } \\
& \text { - miRNA1 }=\text { miRNA6 }
\end{aligned}
$$

Fig. 7 Diversity of short rRFs/miRNAs in Rhodotorula frigidialcoholis and PCoA analysis (Bray-Curtis) of $R$. frigidialcoholis short rRFs/ miRNAs at 0 and $23^{\circ} \mathbf{C}$. A Abundance and diversity of the short rRFs/miRNAs (15-30 nt), with a minimum of 50 reads, of the three $0{ }^{\circ} \mathrm{C}$ and the three $23^{\circ} \mathrm{C} R$. frigidialcoholis cultures. B PCoA analysis (Bray-Curtis) of the three $0^{\circ} \mathrm{C}$ (blue) and the $23^{\circ} \mathrm{C}$ (red) $R$. frigidialcoholis short rRFs/ miRNAs. The legend on the right lists only the ten most abundant short rRFs/miRNAs.

and limiting carbon loss by switching from the citrate cycle to ethanol fermentation. This consequently may increase its survivability in the extremely cold Antarctic permafrost habitat from which it was isolated (Fig. 3).

\section{Small non-coding RNA expression changes under cold temperature}

Small non-coding RNA ( $<200$ nucleotides; sRNA) have numerous functions, including protein synthesis (small rRNAs and tRNAs) and mRNA regulation through RNA interference (RNAi) [102]. The roles of SRNA and RNAi are not well understood, but they have been linked to stress response [103, 104]. Based on quantification of the total RNA extracted from $R$. frigidialcoholis cultures, we initially observed a significantly higher $(p=0.0003)$ proportion of sRNAs $(<200 \mathrm{nt})$ in the $0^{\circ} \mathrm{C}$ compared to $23^{\circ} \mathrm{C}$ cultures (Supplementary Fig. S3). To determine how the sRNA were affected by low temperature in $R$. frigoalcoholis, we further analyzed the composition of sRNAs at 0 and $23^{\circ} \mathrm{C}$. On average, $53.40 \pm 0.99 \%$ of the total RNA was characterized as sRNAs in $0^{\circ} \mathrm{C}$ stressed cultures compared to $27.70 \pm 1.84 \%$ in $23^{\circ} \mathrm{C}$ cultures. There are multiple types of sRNA molecules including miRNAs that are non-coding $\sim 22$ nt sequences that play a role in gene regulation of eukaryote cells, including fungi [105, 106], and rRFs that are derived from rRNA [107]. We subsequently observed a higher proportion $(p=0.029)$ of short rRFs and miRNA (15-30 nt) in R. frigoalcoholis at $0{ }^{\circ} \mathrm{C}$ compared to $23^{\circ} \mathrm{C}$ (Supplementary Fig. S3). Specifically, $0.69 \pm 0.34 \%$ of the total RNA was characterized as short $\mathrm{rRF} / \mathrm{miRNAs}$ in $0^{\circ} \mathrm{C}$ stressed cultures compared to $0.02 \pm 0.01 \%$ at $23^{\circ} \mathrm{C}$, resulting in a $\sim 34$-fold increase at $0^{\circ} \mathrm{C}$. In addition, we observed a higher diversity in short rRF/miRnAs at $0{ }^{\circ} \mathrm{C}$ compared to $23^{\circ} \mathrm{C}$, based on PCoA analysis (Fig. 7 and Supplementary Fig. S3). Stability and non-random sequence distribution of rRFs in previous studies implies that they are not merely products of rRNA turnover, but a functional part of cellular homeostasis, although they have historically been eliminated from sequencing datasets as contaminants [107]. rRFs can be produced from stressed induced cleavage of tRNAs and rRNAs $[107,108]$ and globally downregulate translation by binding to ribosomes $[109,110]$ or by binding to $\mathrm{mRNA}$, both triggering RNAi [107, 111, 112]. Thus, rRF/miRNAs production may be a stress response resulting in overall slower growth rate and metabolism.

Strong links between cold adaptation in prokaryotes and fungi through miRNA regulation have not yet been reported, although this mechanism was identified as a cold adaptation in plants [103]. rRFs have also been linked to cold and UV stress [107], and inhibition of protein translation following DNA damage [113]. Another subset of rRFs are antisense ribosomal small interfering RNAs (risiRNAs) that downregulate pre-rRNA levels through the nuclear RNAi pathway under cold and UV stress to maintain rRNA homeostasis [114, 115]. This phenomenon is triggered by production of erroneous rRNAs transcripts due to environmental stress [116]. Thus, this RNAi pathway potentially decreases global translation by targeting rRNA thus slowing down the cell cycle and limiting production of erroneous proteins.

We could not find any studies clearly reporting the percent of $\mathrm{rRF}$ /miRNAs of total RNA in microorganisms to compare with our results. However, we postulate that the 34 -fold increase of rRF/ miRNAs at $0{ }^{\circ} \mathrm{C}$ in $R$. frigidialcoholis may be a cold temperature response and result from a combination of three possible RNAi mechanisms (Fig. 3): (1) the rRFs were derived from rDNA triggered by DNA damage $[113,115]$ due to cold stress, resulting in global suppression of mRNA translation, and consequently allowing $R$. frigidialcoholis to slow down its cell cycle under cold temperature; (2) the rRFs observed could act via the nuclear RNAi pathway to downregulate pre-rRNA levels thus suppressing global translation via a negative feedback loop that targets immature rRNA. This pathway may be triggered by an increase in errors during rRNA transcription due to environmental stresses such as low temperature [114]. At $0{ }^{\circ} \mathrm{C}$, we observed rRFs with sequence 
Table 1. Characteristic of the ten most abundant $\mathrm{rRF}$ /miRNAs expressed in Rhodotorula frigidialcoholis.

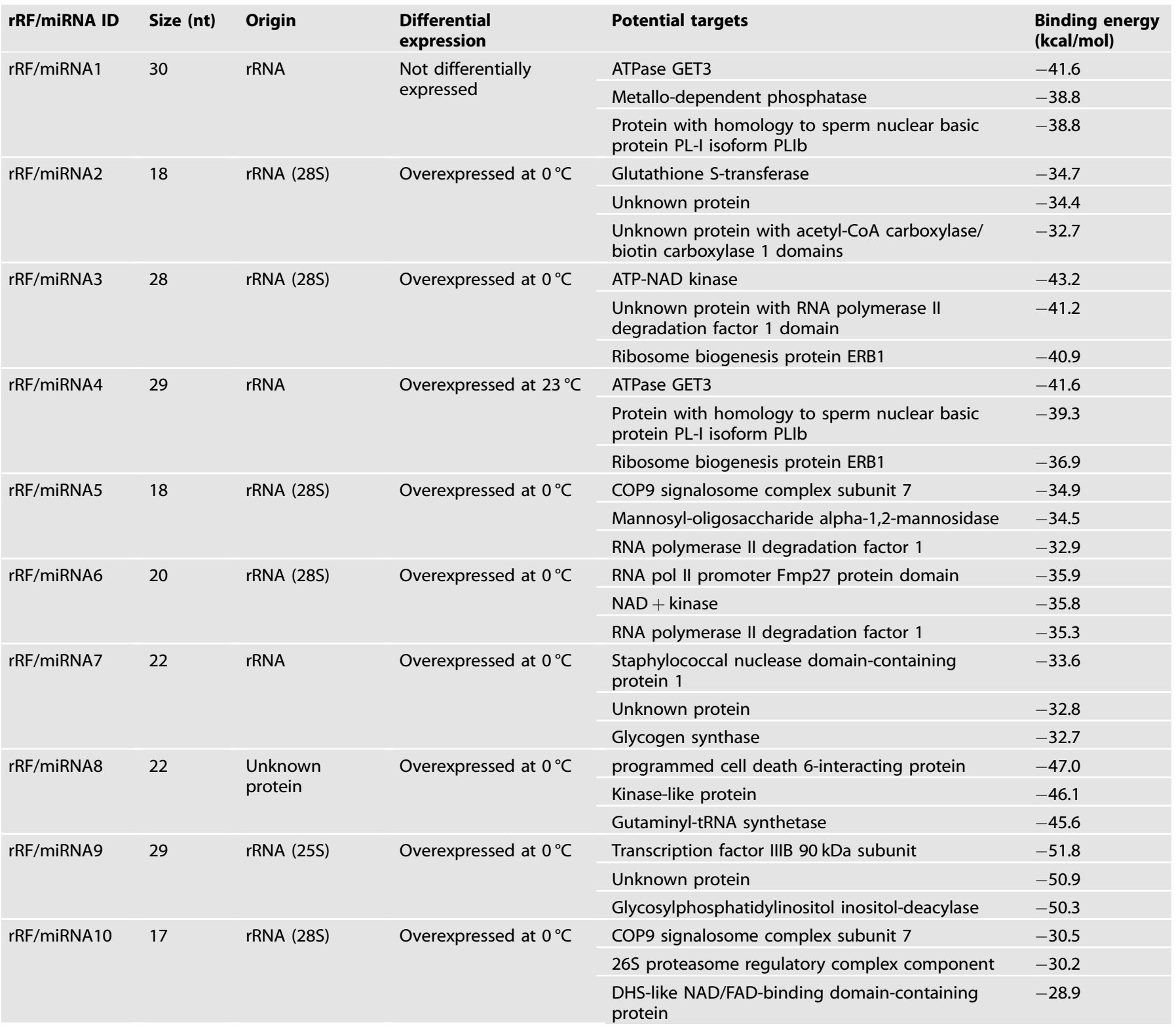

Summary of the transcription origin, and differential expression of $R$. frigidialcoholis miRNAs. Putative genomic origin of the rRFs/miRNAs were predicted with BLAST [38] and their putative mRNA gene targets were predicted using the RNAhybrid computational method [40].

variability suggesting that erroneous rRNA were potentially produced at a higher rate in $R$. frigidialcoholis at $0^{\circ} \mathrm{C}$ than $23^{\circ} \mathrm{C}$. (3) R. frigidialcoholis may contain miRNA derived from mRNA and short rRFs derived from rRNA. Both of these could target and suppress a variety of targeted mRNAs in response to cold, as previously observed in plants [103]. Using a RNAhybrid computational method [40], the top putative mRNA targets for the ten most expressed short rRFs/miRNAs (Table 1) were predicted, based on the lowest energy of hybridization required to form a duplex [106]. Of the ten most abundant short rRFs/miRNAs, nine appear to originate from rRNA and one from a hypothetical protein (Table 1). These short rRFs/miRNAs were mainly overexpressed at $0^{\circ} \mathrm{C}$ in $R$. frigidialcoholis and potentially target a wide variety of mRNAs as summarized in Table 1. However, we could not directly correlate the increase in expression of the short rRFs/ miRNAs with a decrease in expression of their putative mRNA targets, nor do the targets appear to be necessarily linked to cold adaptation. Absence of correlation between specific mRNA target expression levels and short rRFs/miRNAs levels could potentially be due to (a) multiple targets per short rRFs/miRNAs, (b) short rRFs/miRNAs targeting rRNAs (instead of mRNAs) that results in an overall global reduction of translation, or (c) RNAi only partially contributing to gene expression regulation.

Through the action of two RNAse III-type proteins, Drosha and Dicer, miRNAs are produced and recognize specific "target" mRNA that are then silenced via RNAi. Drosha is responsible for the primary transcript cleavage to create precursor miRNA, while Dicer, along with Argonaute (Ago), cleaves the precursor miRNA to form a double strand miRNA. One of these miRNA strands is incorporated into the RNA-induced silencing complex that suppresses translation [106]. Short rRFs have been associated with Ago proteins suggesting that rRFs may act like ribosomal derived miRNA as well [112]. Based on the transcriptomic results, the gene coding for Drosha was not differentially expressed; 
however, Dicer ( $F C=3.83, p=0.01)$ and RNA polymerase III subunits were significantly overexpressed at $0{ }^{\circ} \mathrm{C}$ (Supplementary Table S4). This was consistent with a significantly higher proportion of short rRFs/miRNAs at $0{ }^{\circ} \mathrm{C}$ compared to $23^{\circ} \mathrm{C}$ and a decrease in the transcript levels of the translational machinery (ribosome proteins and tRNAs) at $0{ }^{\circ} \mathrm{C}$ (Fig. 2). Taken together, these results suggest that $R$. frigidialcoholis may induce a short $\mathrm{rRF} / \mathrm{miRNAs}$ gene regulatory mechanism in response to cold that triggers translational repression; however, molecular characterizations of the short rRFs/miRNAs, such as stem-loop RT-qPCR and northern blot analysis, are necessary to confirm the involvement of RNAi in cold adaptation.

\section{CONCLUSIONS}

Transcriptomic analyses results suggest that Rhodotorula frigidialcoholis adapts to cold temperatures in the Antarctic dry valley permafrost through a variety of mechanisms including increasing expression of the PPP genes, increasing the production of carotenoids, sphingolipids, unsaturated fatty acid, and exopolysaccharides while coupled with a reduction in expression of growth, transcriptional and translational machinery genes. We also identified novel cold adaptation features including a switch from respiratory metabolism at $23^{\circ} \mathrm{C}$ to fermentative metabolism when grown in a cold condition $\left(0^{\circ} \mathrm{C}\right)$, via downregulation of citrate cycle genes and the ETC genes. In parallel, $R$. frigidialcoholis overexpressed genes involved in ethanol and xylitol fermentation, resulting in ethanol production at the lowest known temperature described so far in any microorganism. This switch may thus be an adaptation to save energy and delay intracellular freezing in cryoenvironments, such as the Antarctic permafrost. At low temperature, $R$. frigidialcoholis also produced a significantly higher proportion of sRNAs, specifically short rRFs/miRNAs, and overexpressed the Dicer gene, suggesting RNAi as a novel mechanism of cold adaptation in polar fungi. Further characterization of the role of ethanol production and identification of mRNA targets of RNAi are needed to determine the roles they play in cold adaptation of yeasts inhabiting the cryosphere. Taken together, our results indicate that $R$. frigidialcoholis has evolved multiple mechanisms to survive in one of the coldest and driest cold environments on Earth.

\section{REFERENCES}

1. Goordial J, Davila A, Lacelle D, Pollard W, Marinova MM, Greer CW, et al. Nearing the cold-arid limits of microbial life in permafrost of an upper dry valley, Antarctica. ISME J. 2016;10:1613.

2. Mykytczuk NC, Foote SJ, Omelon CR, Southam G, Greer CW, Whyte LG. Bacterial growth at $-15 \mathrm{C}$; molecular insights from the permafrost bacterium Planococcus halocryophilus Or1. ISME J. 2013;7:1211.

3. Margesin R, Miteva V. Diversity and ecology of psychrophilic microorganisms. Res Microbiol. 2011;162:346-61.

4. De Maayer P, Anderson D, Cary C, Cowan DA. Some like it cold: understanding the survival strategies of psychrophiles. EMBO Rep. 2014;15:508-17.

5. Hassan N, Rafiq M, Hayat M, Shah AA, Hasan F. Psychrophilic and psychrotrophic fungi: a comprehensive review. Rev Environ Sci Bio. 2016;15:147-72.

6. Christner BC, Mosley-Thompson E, Thompson LG, Reeve JN. Bacterial recovery from ancient glacial ice. Environ Microbiol. 2003;5:433-6.

7. Raymond-Bouchard I, Goordial J, Zolotarov Y, Ronholm J, Stromvik M, Bakermans $C$, et al. Conserved genomic and amino acid traits of cold adaptation in subzero-growing Arctic permafrost bacteria. FEMS Microbiol Ecol. 2018;94: fiy023.

8. Raymond-Bouchard I, Tremblay J, Altshuler I, Greer CW, Whyte LG. Comparative transcriptomics of cold growth and adaptive features of a eury-and stenopsychrophile. Front Microbiol. 2018;9:1565.

9. Buzzini P, Margesin R. Cold-adapted yeasts: a lesson from the cold and a challenge for the XXI century. In: Buzzini P, Margesin R, editors. Cold-adapted yeasts. Heidelberg: Springer; 2014. p. 3-22.

10. Altshuler I, Goordial J, Whyte LG. Microbial life in permafrost. In: Margesin R, editor. Psychrophiles: from biodiversity to biotechnology. 2nd edn. Cham: Springer; 2017. p. 153-79.
11. Gilichinsky D, Wilson G, Friedmann E, McKay C, Sletten R, Rivkina E, et al. Microbial populations in Antarctic permafrost: biodiversity, state, age, and implication for astrobiology. Astrobiology. 2007;7:275-311.

12. de Menezes GCA, Porto BA, Amorim SS, Zani CL, de Almeida Alves TM, Junior PAS, et al. Fungi in glacial ice of Antarctica: diversity, distribution and bioprospecting of bioactive compounds. Extremophiles. 2020;24:367-76.

13. Zhang $\mathrm{T}$, Wang $\mathrm{N}, \mathrm{Yu}$ L. Soil fungal community composition differs significantly among the Antarctic, Arctic, and Tibetan Plateau. Extremophiles. 2020;24:821-9.

14. Coleine C, Zucconi L, Onofri S, Pombubpa N, Stajich JE, Selbmann L. Sun exposure shapes functional grouping of fungi in cryptoendolithic Antarctic communities. Life. 2018;8:19.

15. Gunde-Cimerman N, Zalar P, de Hoog S, Plemenitaš A. Hypersaline waters in salterns-natural ecological niches for halophilic black yeasts. FEMS Microbiol Ecol. 2000;32:235-40.

16. Perini L, Gostinčar C, Anesio AM, Williamson C, Tranter M, Gunde-Cimerman N. Darkening of the Greenland Ice Sheet: fungal abundance and diversity are associated with algal bloom. Front Microbiol. 2019;10:557.

17. Tojo M, Newsham KK. Snow moulds in polar environments. Fungal Ecol. 2012;5:395-402.

18. Rosa LH, Vaz AB, Caligiorne RB, Campolina S, Rosa CA. Endophytic fungi associated with the Antarctic grass Deschampsia antarctica Desv.(Poaceae). Polar Biol. 2009;32:161-7.

19. Gianoli E, Inostroza P, Zúñiga-Feest A, Reyes-Díaz M, Cavieres LA, Bravo LA, et al. Ecotypic differentiation in morphology and cold resistance in populations of Colobanthus quitensis (Caryophyllaceae) from the Andes of central Chile and the maritime Antarctic. Arct Antarct Alp Res. 2004;36:484-9.

20. Duncan SM, Farrell RL, Thwaites JM, Held BW, Arenz BE, Jurgens JA, et al. Endoglucanase-producing fungi isolated from Cape Evans historic expedition hut on Ross Island, Antarctica. Environ Microbiol. 2006;8:1212-9.

21. Starmer WT, Lachance M-A. Yeast ecology. In: Kurtzman CP, Fell JW, Boekhout T, eds. The yeasts. $5 \mathrm{ft}$ ed. London: Elsevier; 2011. p. 65-83.

22. Shivaji S, Prasad G. Antarctic yeasts: biodiversity and potential applications. In: Satyanarayana T, Kunze G, editors. Yeast biotechnology: diversity and applications. New Delhi: Springer; 2009. p. 3-18.

23. Gunde-Cimerman N, Plemenitaš A, Buzzini P. Changes in lipids composition and fluidity of yeast plasma membrane as response to cold. In: Buzzini P, Margesin R, editors. Cold-adapted yeasts. Heidelberg: Springer; 2014. p. 225-42.

24. Goordial J, Raymond-Bouchard I, Riley R, Ronholm J, Shapiro N, Woyke T, et al. Improved high-quality draft genome sequence of the eurypsychrophile Rhodotorula sp. JG1b, isolated from permafrost in the hyperarid upper-elevation mcmurdo dry valleys, Antarctica. Genome Announc. 2016;4:e00069-16.

25. Yen H-W, Liao Y-T, Liu YX. Cultivation of oleaginous Rhodotorula mucilaginosa in airlift bioreactor by using seawater. J Biosci Bioeng. 2016;121:209-12.

26. Buzzini $P$, Turk $M$, Perini $L$, Turchetti $B$, Gunde-Cimerman N. Yeasts in polar and subpolar habitats. In: Buzzini P, Lachance M-A, Yurkov A, editors. Yeasts in natural ecosystems: diversity. Cham: Springer; 2017. p. 331-65.

27. Margesin R, Fonteyne P-A, Schinner F, Sampaio JP. Rhodotorula psychrophila sp. nov., Rhodotorula psychrophenolica sp. nov. and Rhodotorula glacialis sp. nov., novel psychrophilic basidiomycetous yeast species isolated from alpine environments. Int J Syst Evol Micr. 2007;57:2179-84.

28. Sabri A, Jacques P, Weekers F, Bare G, Hiligsmann S, Moussaif M, et al. Effect of temperature on growth of psychrophilic and psychrotrophic members of Rhodotorula aurantiaca. In: Walt DR, editor. Applied biochemistry and biotechnology. New York: Springer Science+Business Media; 2000. p. 391-9.

29. Marchant DR, Head JW III. Antarctic dry valleys: microclimate zonation, variable geomorphic processes, and implications for assessing climate change on Mars. Icarus 2007;192:187-222.

30. Kurtzman C, Fell JW, Boekhout T, editors. The yeasts: a taxonomic study. $5 \mathrm{ft}$ ed. London: Elsevier; 2011.

31. Kornerup A, Wanscher JH, editors. Methuen handbook of colour. 2nd ed. London: Methuen and Co.; 1967.

32. Xing W, Yin M, Lv Q, Hu Y, Liu C, Zhang J. Oxygen solubility, diffusion coefficient, and solution viscosity. In: Xing W, Yin G, Zhang J, editors. Rotating electrode methods and oxygen reduction electrocatalysts. London: Elsevier; 2014. p. 1-31.

33. Viti C, Decorosi F, Marchi E, Galardini M, Giovannetti L. High-throughput phenomics. In: Mengoni A, Galardini M, Fondi M, editors. Bacterial pangenomics. Methods and protocols. New York: Springer; 2015. p. 99-123.

34. Rico A, Preston GM. Pseudomonas syringae pv. tomato DC3000 uses constitutive and apoplast-induced nutrient assimilation pathways to catabolize nutrients that are abundant in the tomato apoplast. Mol Plant Microbe. 2008;21:269-82.

35. Patro R, Duggal G, Love MI, Irizarry RA, Kingsford C. Salmon provides fast and bias-aware quantification of transcript expression. Nat Methods. 2017;14:417.

36. Love MI, Huber W, Anders S. Moderated estimation of fold change and dispersion for RNA-seq data with DESeq2. Genome Biol. 2014;15:550. 
37. Kanehisa M, Sato Y, Morishima K. BlastKOALA and GhostKOALA: KEGG tools for functional characterization of genome and metagenome sequences. J Mol Biol. 2016;428:726-31.

38. Altschul SF, Gish W, Miller W, Myers EW, Lipman DJ. Basic local alignment search tool. J Mol Biol. 1990;215:403-10.

39. Krüger J, Rehmsmeier M. RNAhybrid: microRNA target prediction easy, fast and flexible. Nucleic Acids Res. 2006;34:W451-54.

40. Rehmsmeier $M$, Steffen $P$, Höchsmann $M$, Giegerich R. Fast and effective prediction of microRNA/target duplexes. RNA. 2004;10:1507-17.

41. Greetham D. Phenotype microarray technology and its application in industrial biotechnology. Biotechnol Lett. 2014;36:1153-60.

42. Bochner BR. Global phenotypic characterization of bacteria. FEMS Microbiol Rev. 2008;33:191-205.

43. Maldonado F, Packard T, Gómez M. Understanding tetrazolium reduction and the importance of substrates in measuring respiratory electron transport activity. J Exp Mar Biol Ecol. 2012;434:110-8.

44. Barclay BJ, DeHaan CL, Hennig UG, lavorovska O, von Borstel RW, Von, et al. A rapid assay for mitochondrial DNA damage and respiratory chain inhibition in the yeast Saccharomyces cerevisiae. Environ Mol Mutagen. 2001;38:153-8.

45. Jenkins CL, Lawrence SJ, Kennedy Al, Thurston P, Hodgson JA, Smart KA. Incidence and formation of petite mutants in lager brewing yeast Saccharomyces cerevisiae (syn. S. pastorianus) populations. J Am Soc Brew Chem 2009;67:72-80.

46. Glab N, Wise R, Pring D, Jacq C, Slonimski P. Expression in Saccharomyces cerevisiae of a gene associated with cytoplasmic male sterility from maize: respiratory dysfunction and uncoupling of yeast mitochondria. Mol Gen Genet. 1990;223:24-32.

47. Goldring ES, Grossman LI, Krupnick D, Cryer DR, Marmur J. The petite mutation in yeast: loss of mitochondrial deoxyribonucleic acid during induction of petites with ethidium bromide. J Mol Biol. 1970;52:323-35.

48. Vogel C, Marcotte EM. Insights into the regulation of protein abundance from proteomic and transcriptomic analyses. Nat Rev Genet. 2012;13:227-32.

49. Pinatel $E$, Peano $C$. RNA sequencing and analysis in microorganisms for metabolic network reconstruction. In: Fondi $M$, editor. Metabolic network reconstruction and modeling. Methods and protocols. New York: Springer; 2018. p. 239-65.

50. Raymond-Bouchard I, Chourey K, Altshuler I, lyer R, Hettich RL, Whyte LG. Mechanisms of subzero growth in the cryophile Planococcus halocryophilus determined through proteomic analysis. Environ Microbiol. 2017;19:4460-79.

51. Bhuiyan M, Tucker D, Watson K. Gas chromatography-mass spectrometry analysis of fatty acid profiles of Antarctic and non-Antarctic yeasts. Anton Leeuw. 2014;106:381-9.

52. López-Malo M, Chiva R, Rozes N, Guillamon JM. Phenotypic analysis of mutant and overexpressing strains of lipid metabolism genes in Saccharomyces cerevisiae: implication in growth at low temperatures. Int J Food Microbiol. 2013;162:26-36

53. Rossi M, Buzzini P, Cordisco L, Amaretti A, Sala M, Raimondi S, et al. Growth, lipid accumulation, and fatty acid composition in obligate psychrophilic, facultative psychrophilic, and mesophilic yeasts. FEMS Microbiol Ecol. 2009;69:363-72.

54. Contreras G, Barahona S, Sepúlveda D, Baeza M, Cifuentes V, Alcaíno J. Identification and analysis of metabolite production with biotechnological potential in Xanthophyllomyces dendrorhous isolates. World J Micro Biot. 2015;31:517-26.

55. Libkind D, Arts M, Van Broock M. Fatty acid composition of cold-adapted carotenogenic basidiomycetous yeasts. Rev Argent Microbiol. 2008;40:193-7.

56. Thomas-Hall S, Watson K. Cryptococcus nyarrowii sp. nov., a basidiomycetous yeast from Antarctica. Int J Syst Evol Micr. 2002;52:1033-8.

57. López-Malo M, García-Ríos E, Chiva R, Guillamon JM. Functional analysis of lipid metabolism genes in wine yeasts during alcoholic fermentation at low temperature. Micro Cell. 2014;1:365.

58. Tai SL, Daran-Lapujade P, Walsh MC, Pronk JT, Daran J-M. Acclimation of SaCcharomyces cerevisiae to low temperature: a chemostat-based transcriptome analysis. Mol Biol Cell. 2007;18:5100-12.

59. Mao C, Wadleigh M, Jenkins GM, Hannun YA, Obeid LM. Identification and characterization of Saccharomyces cerevisiae dihydrosphingosine-1-phosphate phosphatase. J Biol Chem. 1997;272:28690-4.

60. Mata-Gómez LC, Montañez JC, Méndez-Zavala A, Aguilar CN. Biotechnological production of carotenoids by yeasts: an overview. Micro Cell Fact. 2014;13:12.

61. Moliné $M$, Flores MR, Libkind D. del Carmen Diéguez M, Farías ME, van Broock M. Photoprotection by carotenoid pigments in the yeast Rhodotorula mucilaginosa: the role of torularhodin. Photoch Photobio Sci. 2010;9:1145-51.

62. Liu GY, Nizet V. Color me bad: microbial pigments as virulence factors. Trends Microbiol. 2009;17:406-13.

63. Rodrigues DF, Tiedje JM. Coping with our cold planet. Appl Environ Micro. 2008;74:1677-86.
64. Villarreal P, Carrasco M, Barahona S, Alcaíno J, Cifuentes V, Baeza M. Tolerance to ultraviolet radiation of psychrotolerant yeasts and analysis of their carotenoid, mycosporine, and ergosterol content. Curr Microbiol. 2016;72:94-101.

65. Moliné $M$, Libkind $D$, del Carmen DiéguezM, van Broock M. Photoprotective role of carotenoids in yeasts: response to UV-B of pigmented and naturally-occurring albino strains. J Photoch Photobio B 2009;95:156-61.

66. Huang G-T, Ma S-L, Bai L-P, Zhang L, Ma H, Jia P, et al. Signal transduction during cold, salt, and drought stresses in plants. Mol Biol Rep. 2012;39:969-87.

67. Heino P, Palva ET. Signal transduction in plant cold acclimation. In: Hirt H, Shinozaki K, editors. Plant responses to abiotic stress. Berlin: Springer; 2003. p. 151-86.

68. Storey KB, Storey JM. Signal transduction and gene expression in the regulation of natural freezing survival. In: Storey KB, Storey JM, editors. Protein adaptations and signal transduction. London: Elsevier; 2001. p. 1-19.

69. Li W-H, Yang J, Gu X. Expression divergence between duplicate genes. Trends Genet. 2005;21:602-7.

70. Vollmers J, Voget S, Dietrich S, Gollnow K, Smits M, Meyer K, et al. Poles apart: arctic and Antarctic Octadecabacter strains share high genome plasticity and a new type of xanthorhodopsin. Plos One. 2013;8:e63422.

71. Wagner A. Asymmetric functional divergence of duplicate genes in yeast. Mol Biol Evol. 2002;19:1760-8.

72. Varki A, Gagneux P. Biological functions of glycans. In: Varki A, Cummings RD, Esko JD, Stanley P, Hart GW, Aebi M, et al. editors. Essentials of glycobiology. 3rd ed. Cold Spring Harbor: Cold Spring Harbor Laboratory Press; 2017.

73. Colley K, Varki A, Kinoshita T. Cellular organization of glycosylation. In: Varki A, Cummings RD, Esko JD, Stanley P, Hart GW, Aebi M, et al. editors. Essentials of glycobiology. 3rd ed. Cold Spring Harbor: Cold Spring Harbor Laboratory Press; 2017.

74. Pavlova K, Panchev I, Hristozova T. Physico-chemical characterization of exomannan from Rhodotorula acheniorum MC. World J Micro Biot. 2005;21:279-83.

75. Cho DH, Chae HJ, Kim EY. Synthesis and characterization of a novel extracellular polysaccharide by Rhodotorula glutinis. Appl Biochem Biotech. 2001;95:183-93.

76. Flemming HC, Neu TR, Wingender J. The perfect slime. Microbial extracellular polymeric substances (EPS). London: IWA Publishing; 2016.

77. Nichols WW, Evans MJ, Slack MP, Walmsley HL. The penetration of antibiotics into aggregates of mucoid and non-mucoid Pseudomonas aeruginosa. Microbiology. 1989;135:1291-303.

78. Selbmann L, Onofri S, Fenice M, Federici F, Petruccioli M. Production and structural characterization of the exopolysaccharide of the Antarctic fungus Phoma herbarum CCFEE 5080. Res Microbiol. 2002;153:585-92.

79. Rini JM, Esko JD. Glycosyltransferases and glycan-processing enzymes. In: Varki A, Cummings RD, Esko JD, Stanley P, Hart GW, Aebi M, et al. editors. Essentials of glycobiology. 3rd ed. Cold Spring Harbor: Cold Spring Harbor Laboratory Press; 2017.

80. Strassburg K, Walther D, Takahashi H, Kanaya S, Kopka J. Dynamic transcriptional and metabolic responses in yeast adapting to temperature stress. Omics. 2010;14:249-59.

81. Becerra $M$, Lombardia L, Gonzalez-Siso M, Rodriguez-Belmonte $E$, Hauser N, Cerdán M. Genome-wide analysis of the yeast transcriptome upon heat and cold shock. Int J Genomics. 2003;4:366-75.

82. Homma $\mathrm{T}$, Iwahashi $\mathrm{H}$, Komatsu $\mathrm{Y}$. Yeast gene expression during growth at low temperature. Cryobiology. 2003;46:230-7.

83. Sahara T, Goda T, Ohgiya S. Comprehensive expression analysis of timedependent genetic responses in yeast cells to low temperature. J Biol Chem. 2002;277:50015-21.

84. Schade B, Jansen G, Whiteway M, Entian KD, Thomas DY. Cold adaptation in budding yeast. Mol Biol Cell. 2004;15:5492-502.

85. Mikami K, Kanesaki Y, Suzuki I, Murata N. The histidine kinase Hik33 perceives osmotic stress and cold stress in Synechocystis sp. PCC 6803. Mol Microbiol. 2002;46:905-15.

86. Tsuji M. Cold-stress responses in the Antarctic basidiomycetous yeast Mrakia blollopis. R Soc Open Sci. 2016;3:160106.

87. Sarkar D, Bhowmik PC, Kwon Y-I, Shetty K. Clonal response to cold tolerance in creeping bentgrass and role of proline-associated pentose phosphate pathway. Bioresour Technol. 2009;100:5332-9.

88. Bura R, Vajzovic A, Doty SL. Novel endophytic yeast Rhodotorula mucilaginosa strain PTD3 I: production of xylitol and ethanol. J Ind Microbiol Biot. 2012;39:1003-11.

89. da Silva TL, Feijão D, Roseiro JC, Reis A. Monitoring Rhodotorula glutinis CCMI 145 physiological response and oil production growing on xylose and glucose using multi-parameter flow cytometry. Bioresour Technol. 2011;102:2998-3006.

90. Johansson B, Hahn-Hägerdal B. The non-oxidative pentose phosphate pathway controls the fermentation rate of xylulose but not of xylose in Saccharomyces cerevisiae TMB3001. FEMS Yeast Res. 2002;2:277-82. 
91. Eliasson A, Boles E, Johansson B, Österberg M, Thevelein J, Spencer-Martins I, et al. Xylulose fermentation by mutant and wild-type strains of Zygosaccharomyces and Saccharomyces cerevisiae. Appl Microbiol Biot. 2000;53:376-82.

92. Mohamad N, Mustapa Kamal S, Mokhtar M. Xylitol biological production: a review of recent studies. Food Rev Int. 2015;31:74-89.

93. Shetty K, Wahlqvist M. A model for the role of the proline-linked pentosephosphate pathway in phenolic phytochemical bio-synthesis and mechanism of action for human health and environmental applications. Asia Pac J Clin Nutr. 2004;13:1-24.

94. Fonseca P, Moreno R, Rojo F. Growth of Pseudomonas putida at low temperature: global transcriptomic and proteomic analyses. Environ Microbiol Rep. 2011;3:329-39.

95. Rao R, Bhadra B, Shivaji S. Isolation and characterization of ethanol-producing yeasts from fruits and tree barks. Lett Appl Microbiol. 2008;47:19-24.

96. Kourkoutas Y, Komaitis M, Koutinas A, Kaliafas A, Kanellaki M, Marchant R, et al. Wine production using yeast immobilized on quince biocatalyst at temperatures between 30 and 0 C. Food Chem. 2003;82:353-60.

97. Kanellaki M, Koutinas AA. Low temperature fermentation of wine and beer by cold-adapted and immobilized yeast cells. In: Margesin R, Schinner F, editors. Biotechnological applications of cold-adapted organisms. Berlin: Springer; 1999. p. $117-45$.

98. Bakoyianis V, Kanellaki M, Kaliafas A, Koutinas A. Low-temperature wine making by immobilized cells on mineral kissiris. J Agr Food Chem. 1992;40:1293-6.

99. Tiwari R, Singh S, Shukla P, Nain L. Novel cold temperature active $\beta$-glucosidase from Pseudomonas lutea BG8 suitable for simultaneous saccharification and fermentation. RSC Adv. 2014;4:58108-15.

100. Tang W, Wang Y, Zhang J, Cai Y, He Z. Biosynthetic pathway of carotenoids in Rhodotorula and strategies for enhanced their production. J Microbiol Biotechn. 2019;29:507-17.

101. Steven B, Briggs G, McKay CP, Pollard WH, Greer CW, Whyte LG. Characterization of the microbial diversity in a permafrost sample from the Canadian high Arctic using culture-dependent and culture-independent methods. FEMS Microbiol Ecol. 2007;59:513-23.

102. Dozmorov MG, Giles CB, Koelsch KA, Wren JD. Systematic classification of noncoding RNAs by epigenomic similarity. BMC Bioinforma. 2013;14:S2.

103. Sunkar R, Li Y-F, Jagadeeswaran G. Functions of microRNAs in plant stress responses. Trends Plant Sci. 2012;17:196-203.

104. Ambros V. MicroRNA pathways in flies and worms: growth, death, fat, stress, and timing. Cell. 2003;113:673-6.

105. Lau SK, Chow W-N, Wong AY, Yeung JM, Bao J, Zhang N, et al. Identification of microRNA-like RNAs in mycelial and yeast phases of the thermal dimorphic fungus Penicillium marneffei. Plos Negl Trop D. 2013;7:e2398.

106. Zhou Q, Wang Z, Zhang J, Meng H, Huang B. Genome-wide identification and profiling of microRNA-like RNAs from Metarhizium anisopliae during development. Fungal Biol UK. 2012;116:1156-62.

107. Lambert M, Benmoussa A, Provost P. Small non-coding RNAs derived from eukaryotic ribosomal RNA. Noncoding RNA 2019;5:16.

108. Thompson DM, Lu C, Green PJ, Parker R. tRNA cleavage is a conserved response to oxidative stress in eukaryotes. RNA. 2008;14:2095-103.

109. Gebetsberger J, Wyss L, Mleczko AM, Reuther J, Polacek N. A tRNA-derived fragment competes with mRNA for ribosome binding and regulates translation during stress. RNA Biol. 2017;14:1364-73.

110. Bąkowska-Żywicka K, Kasprzyk M, Twardowski T. tRNA-derived short RNAs bind to Saccharomyces cerevisiae ribosomes in a stress-dependent manner and inhibit protein synthesis in vitro. FEMS Yeast Res. 2016;16:fow077.

111. McCool MA, Bryant CJ, Baserga SJ. MicroRNAs and long non-coding RNAs as novel regulators of ribosome biogenesis. Biochem Soc T. 2020;48:595-612.

112. Wei H, Zhou B, Zhang F, Tu Y, Hu Y, Zhang B, et al. Profiling and identification of small rDNA-derived RNAs and their potential biological functions. Plos One. 2013;8:e56842.

113. Lee H-C, Chang S-S, Choudhary S, Aalto AP, Maiti M, Bamford DH, et al. qiRNA is a new type of small interfering RNA induced by DNA damage. Nature. 2009;459:274-7.
114. Zhu C, Yan Q, Weng C, Hou X, Mao H, Liu D, et al. Erroneous ribosomal RNAs promote the generation of antisense ribosomal siRNA. P Natl Acad Sci USA. 2018; 115:10082-7.

115. Zhou X, Chen X, Wang Y, Feng X, Guang S. A new layer of rRNA regulation by small interference RNAs and the nuclear RNAi pathway. RNA Biol. 2017;14:1492-8.

116. Zhou X, Feng X, Mao H, Li M, Xu F, Hu K, et al. RdRP-synthesized antisense ribosomal siRNAs silence pre-rRNA via the nuclear RNAi pathway. Nat Struct Mol Biol. 2017;24:258

\section{ACKNOWLEDGEMENTS}

The authors acknowledge the valuable input from $\mathrm{Dr}$ Jennifer Ronholm and $\mathrm{Dr}$ Jacqueline Goordial for their critical feedback and for the setting up of the project, and Dr Barry R. Bochner, from Biolog Inc. for his valuable advice with the Biolog Phenotypic MicroArray tests analysis. The authors acknowledge the support of the McGill Space Institute.

\section{AUTHOR CONTRIBUTIONS}

DT wrote the manuscript, performed all laboratory experiments and data analysis. IA helped with the experimental design, laboratory experiments, data analysis, figure creation, writing and manuscript editing. CG performed the bioinformatic pipeline analysis of the raw RNASeq data (mRNA). PZ and JZ described the novelty of the species. IR-B helped with experimental design and manuscript editing. LGW and NG-C provided guidance, advise, and helped with the manuscript editing.

\section{COMPETING INTERESTS}

The authors declare no competing interests.

\section{ADDITIONAL INFORMATION}

Supplementary information The online version contains supplementary material available at https://doi.org/10.1038/s41396-021-01030-9.

Correspondence and requests for materials should be addressed to L.G.W.

Reprints and permission information is available at http://www.nature.com/ reprints

Publisher's note Springer Nature remains neutral with regard to jurisdictional claims in published maps and institutional affiliations.

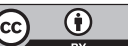

Open Access This article is licensed under a Creative Commons Attribution 4.0 International License, which permits use, sharing, adaptation, distribution and reproduction in any medium or format, as long as you give appropriate credit to the original author(s) and the source, provide a link to the Creative Commons license, and indicate if changes were made. The images or other third party material in this article are included in the article's Creative Commons license, unless indicated otherwise in a credit line to the material. If material is not included in the article's Creative Commons license and your intended use is not permitted by statutory regulation or exceeds the permitted use, you will need to obtain permission directly from the copyright holder. To view a copy of this license, visit http://creativecommons. org/licenses/by/4.0/.

(c) The Author(s) 2021 